\title{
Sensitivity of CTA to gamma-ray emission from the Perseus galaxy cluster
}

\author{
Judit Pérez-Romero ${ }^{a, b, *}$ on behalf of the CTA Consortium \\ ${ }^{a}$ Instituto de Física Teórica UAM-CSIC, Universidad Autónoma de Madrid, C/ Nicolás Cabrera, 13-15, \\ 28049 Madrid, Spain \\ ${ }^{b}$ Departamento de Física Teórica, M-15, Universidad Autónoma de Madrid, E-28049 Madrid, Spain \\ E-mail: judit.perez@uam.es
}

In these proceedings we summarize the current status of the study of the sensitivity of the Cherenkov Telescope Array (CTA) to detect diffuse gamma-ray emission from the Perseus galaxy cluster. Gamma-ray emission is expected in galaxy clusters both from interactions of cosmic rays (CR) with the intra-cluster medium, or as a product of annihilation or decay of dark matter (DM) particles in case they are weakly interactive massive particles (WIMPs). The observation of Perseus constitutes one of the Key Science Projects to be carried out by the CTA Consortium. In this contribution, we focus on the DM-induced component of the flux. Our DM modelling includes the substructures we expect in the main halo which will boost the annihilation signal significantly. We adopt an ON/OFF observation strategy and simulate the expected gamma-ray signals. Finally we compute the expected CTA sensitivity using a likelihood maximization analysis including the most recent CTA instrument response functions. In absence of signal, we show that CTA will allow us to provide stringent and competitive constraints on TeV DM, especially for the case of DM decay.

$37^{\text {th }}$ International Cosmic Ray Conference (ICRC 2021)

July 12th - 23rd, 2021

Online - Berlin, Germany

\footnotetext{
${ }^{*}$ Presenter
} 


\section{Introduction}

Galaxy clusters are the largest gravitationally-bound objects in the Universe, with masses between $M_{200} \approx 10^{14}-10^{15} \mathrm{M}_{\odot}$. Dark matter $(\mathrm{DM})$ is expected to account for about $80 \%$ of their mass [1], while the rest is baryonic matter in the form of galaxies, gas and dust in the intra-cluster medium (ICM). Although clusters are supposedly virialized objects, the presence of hot gas, strong magnetic fields, galaxies and Active Galactic Nuclei (AGNs) produces turbulence phenomena and complex baryonic feedback in the ICM. All these astrophysical processes act as acceleration mechanisms, leading to production of cosmic rays (CR). The presence of CRs has been confirmed through observations of diffuse synchrotron emission produced by the CRs electrons and positrons at different wavelengths [2]. However, the gamma-ray emission, expected either from neutral pion decay from accelerated CRs [3] and/or from DM annihilation or decay of Weakly Interacting Massive Particles (WIMPs) particles [4], has so far avoided detection [5].

Galaxy clusters are considered excellent and complementary targets for gamma-ray DM searches because of the large amount of DM they are expected to host. Given the large number of known clusters, it is key to determine which of them meet the most appropriate conditions to be searched for in gamma-rays:

- Proximity to Earth $(z<0.1)$, so that substantial DM-induced fluxes are expected.

- High-mass clusters with well-determined masses, since the annihilation flux is proportional to the mass.

- Low gamma-ray flux from conventional astrophysical processes [6].

In [7] authors studied how the most promising galaxy clusters compared to the known dwarf Spheroidal galaxies (dSphs) in terms of their annihilation fluxes. Following results of DM-only $\mathrm{N}$-body simulations, the substructures in the main DM halo where for the first time included for the computation of the annihilation flux. This substructures, usually called subhalos, are a natural prediction within the standard $\Lambda \mathrm{CDM}$ structure formation scenario. The authors concluded that galaxy clusters are competitive targets in terms of the annihilation flux, as long as the DM annihilation happening in these subhalos is properly taken into account.

The Cherenkov Telescope Array (CTA) will be the next generation of Imaging Atmospheric Cherenkov Telescopes (IACTs). CTA's energy sensitivity will improve up to one order of magnitude the sensitivity of current generation IACTs in the $20 \mathrm{GeV}$ to $300 \mathrm{TeV}$ energy range [8], will have 2.5 better field of view and 5 times better angular resolution. CTA will have two different arrays: the Northern array in La Palma (Spain), and the Southern array in the Atacama desert in Chile. Having an excellent spectral and angular resolution, CTA has superb capabilities to perform gamma-ray DM searches, especially those focused on WIMPs.

Among the local clusters that fulfill the requirements mentioned before, the Perseus cluster is the brightest in the X-ray sky. It hosts two central AGNs, NGC1275 and IC310. Being one of the most massive clusters in the nearby Universe, Perseus is one of the most promising clusters to be detected in gamma-rays [9]. More precisely, given its position in the sky, it meets the optimal conditions for observation by the CTA Northern array [8]. All these attributes make the Perseus science case an excellent discovery opportunity for CTA [8]. 


\section{Dark Matter modelling in Perseus}

The expected annihilation flux in gamma-rays from the Perseus cluster can be computed as follows [10]:

$$
\frac{d \Phi_{\gamma}}{d E}\left(\Delta \Omega, \text { l.o.s,E) }=\frac{d \phi_{\gamma}}{d E}(E) \times J(\Delta \Omega, \text { l.o.s }),\right.
$$

where $\frac{d \Phi_{\gamma}}{d E}$ is the gamma-ray annihilation/decay flux, $\frac{d \phi_{\gamma}}{d E}$ is the particle physics term containing the spectral information [4] and $J$ is the so-called astrophysical factor. The particle physics term can be written, assuming Majorana WIMPs, in terms of the selected annihilation/decay channel $\left(d N^{P P} / d E\right)$, the mass $\left(m_{D M}\right)$ and the averaged annihilation cross section $(<\sigma v>)$ or the DM particle lifetime $\left(\tau_{D M}\right)$ as

$$
\begin{gathered}
\frac{d \phi_{\gamma}^{\text {Annihil }}}{d E}=\frac{<\sigma v>}{8 \pi m_{D M}^{2}} \frac{d N^{P P}}{d E}, \\
\frac{d \phi_{\gamma}^{\text {Decay }}}{d E}=\frac{1}{4 \pi m_{D M} \tau_{D M}} \frac{d N^{P P}}{d E} .
\end{gathered}
$$

The astrophysical factor or J-factor (D- for decaying DM) accounts for the DM distribution in the halo, parametrized in the DM density profile $\rho_{D M}$ :

$$
\begin{aligned}
& J(\Delta \Omega, \text { l.o.s })=\int_{\Delta \Omega} \int_{\text {l.o.s }} \rho_{D M}^{2}, \\
& D(\Delta \Omega, \text { l.o.s })=\int_{\Delta \Omega} \int_{\text {l.o.s }} \rho_{D M} .
\end{aligned}
$$

From the X-ray surface brightness data of the Perseus galaxy cluster, one can derive the cluster's main parameters. We build the DM density profile for the main halo starting from its measured mass. In our study, we adopt the mass estimate in [11]. We also need to assume a DM profile, in this case, the Navarro-Frenk-White (NFW) profile [12, 13]:

$$
\rho_{\mathrm{NFW}}(r)=\frac{\rho_{0}}{\left(\frac{r}{r_{s}}\right)\left(1+\frac{r}{r_{s}}\right)^{2}} .
$$

The two parameters in the NFW profile are computed by making use of the concentration-mass $(c-M)$ relation in [14]. The obtained DM density profile parameters for Perseus are given in Table 1.

\begin{tabular}{|c|c|c|c|c|c|c|}
\hline Cluster & $\begin{array}{c}(l, b) \\
{[\mathrm{deg}]}\end{array}$ & $\begin{array}{c}D \\
{[\mathrm{Mpc}]}\end{array}$ & $\begin{array}{c}M_{200} \\
{\left[10^{14} \mathrm{M}_{\odot}\right]}\end{array}$ & $\begin{array}{c}R_{200} \\
{\left[10^{2} \mathrm{kpc}\right]}\end{array}$ & $\begin{array}{c}\rho_{0} \\
{\left[10^{6} \mathrm{M}_{\odot} \mathrm{kpc}^{-3}\right]}\end{array}$ & $\begin{array}{c}r_{\mathrm{s}} \\
{\left[10^{2} \mathrm{kpc}\right]}\end{array}$ \\
\hline Perseus & $(150.57,-13.26)$ & 75.01 & 7.52 & 18.65 & 1.20 & 3.71 \\
\hline
\end{tabular}

Table 1: DM density profile parameters for the Perseus galaxy cluster assuming the NFW profile and the $c-M$ relation in [14]. 
We also include in the modelling the expected substructures that contribute to the DM-induced gamma-ray flux. We note that this is only relevant for the annihilation case. We assume an NFW density profile also to describe the internal structure of subhalos. We parametrize the population of subhalos inside the main halo via three basic factors: radial distribution (SRD), mass distribution (SHMF) and subhalo concentration. Following the state-of-the-art of the subhalo DM population as given by $\mathrm{N}$-body simulations, we will adopt:

- SRD: Anti-biased distribution following the results of the Via Lactea - II Milky-Way-sized cosmological simulation [15].

- SHMF: $\frac{d N}{d M} \propto M^{-\alpha}$, where $\alpha$ can take two values $\alpha=1.9,2.0$; following [16] and [15], respectively.

- Concentration: We use the parametrization specifically derived for subhalos developed in [17].

In our work, we define three different models of the subhalo population in Perseus. With the definition of these three models, we will be able to provide an estimate of the uncertainty on the exact configuration of halo substructure in Perseus. Indeed, we will obtain conservative values (no substructure model) for the DM annihilation flux and also upper bounds ( $\alpha=2.0$ model). To compute the J- and D- factors we use the CLUMPY free software [18-20]. The results of the DM modelling for annihilation and decay are shown in Table 2 and the corresponding differential annihilation flux profiles are represented in Figure 1.

\begin{tabular}{|c|c||c|c|}
\hline Annihilation & $\log _{10} J\left[\mathrm{GeV}^{2} \mathrm{~cm}^{-5}\right]$ & Decay & $\log _{10} D\left[\mathrm{GeV} \mathrm{cm}{ }^{-2}\right]$ \\
\hline$J_{T}$ & 17.42 & & \\
\hline$J_{T}^{1.9}$ & 18.48 & $D_{T}$ & 19.20 \\
\hline$J_{T}^{2.0}$ & 18.93 & & \\
\hline
\end{tabular}

Table 2: Annihilation of the different DM models (no substructure, SHMF $\alpha=1.9$, SHMF $\alpha=2.0$ ) and decay results for Perseus, integrated up to $R_{200}$ (symbolized as the sub-index $T$ ). See text for for details.
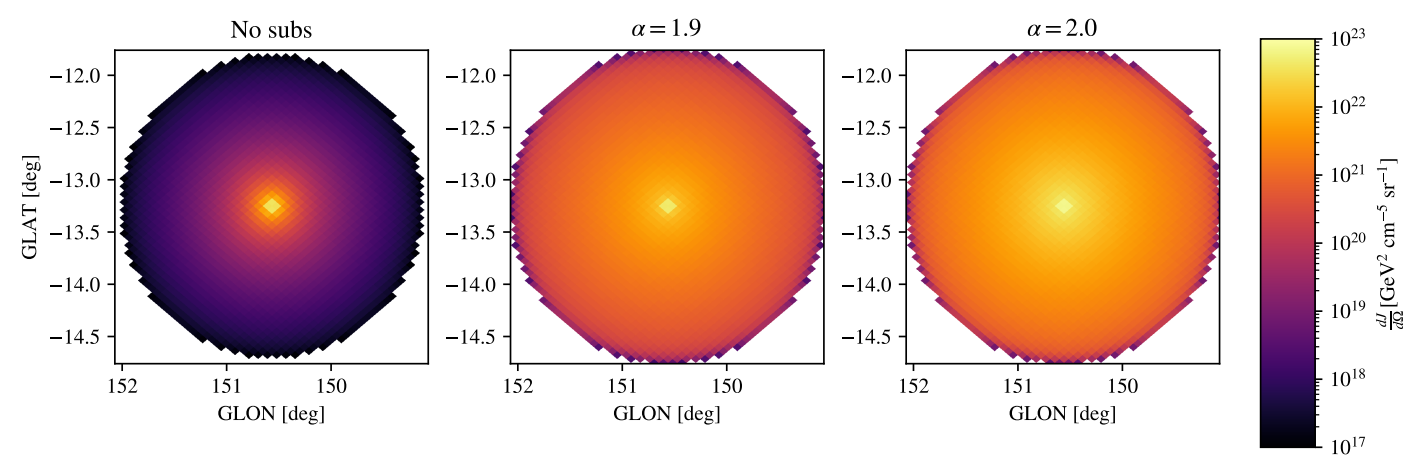

Figure 1: Two-dimensional spatial templates showing the expected spatial morphology of the DM annhilation flux in Perseus. The inclusion of different amounts of substructure as described in the text alters the flux especially in the outskirts of the cluster. 
From the J-factor values of Table 2 we can notice the effect of taking into account the substructure in the cluster. This enhancement of the J-factor when including the subhalos in the DM modelling is known as the subhalo boost factor $B$, defined as $B=0$ for the case where no subestructure is included. For the model with $\alpha=1.9$ the boost factor is $B=10.5$, and for $\alpha=2.0$ we obtain $B=31.4$.

\section{CTA sensitivity to DM-induced gamma-ray from Perseus}

We use the most up-to-date Instrument Response Functions (IRFs - prod3b-v2) to compute the prospects for observing gamma-rays from Perseus using CTA. Besides, we also use the two available softwares to perform the analysis, gammapy ${ }^{1}$ and ctools ${ }^{2}$. In our analysis, we include DM annihilation/decay as gamma-ray source. In future steps of the project, we will consider also CR-induced gamma-ray emission and the emission from the two AGNs hosted in Perseus. An illustrative sketch of the analysis pipeline to follow in our work is shown in Figure 2.

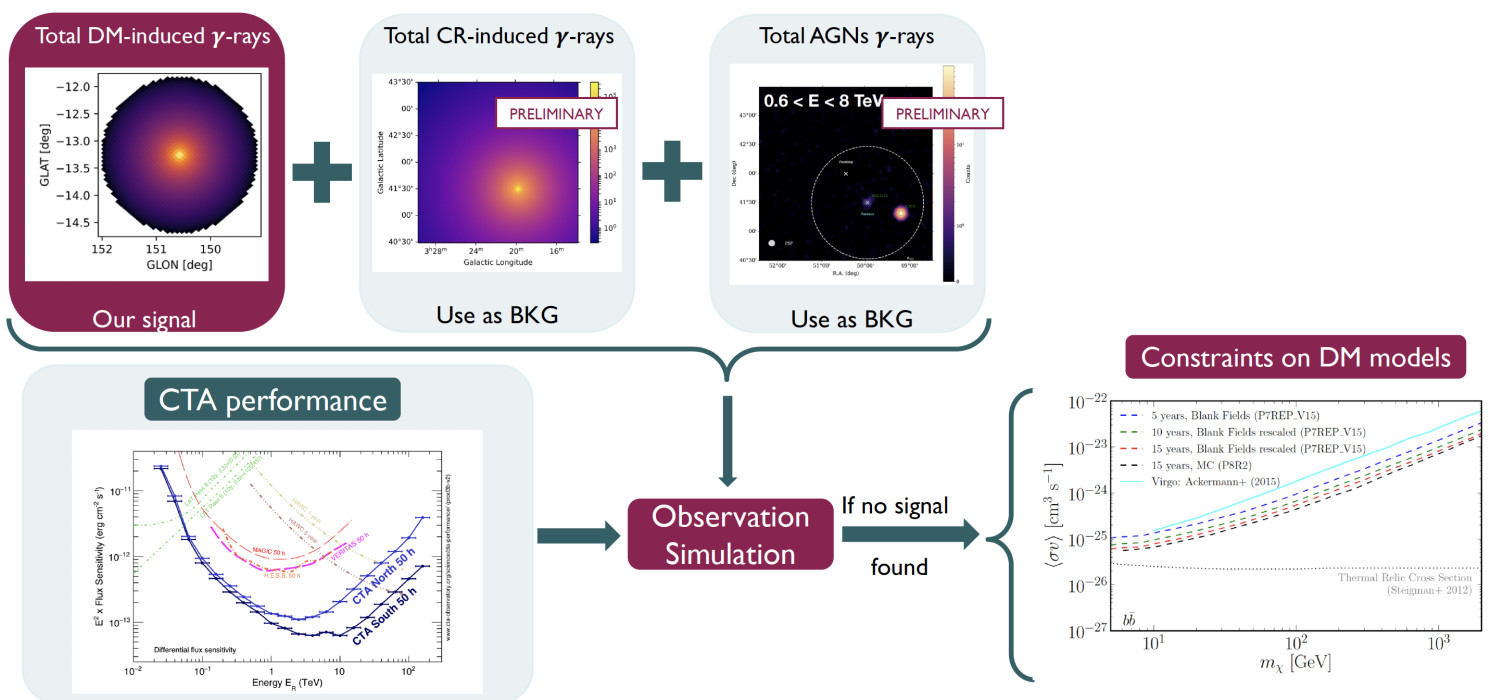

Figure 2: Sketch of the analysis pipeline to compute the sensitivity of CTA to gamma-ray induced DM signal. The bottom right plot is only shown as an example of constraints from this type of searches, and was extracted from [21].

The analysis strategy is based on the widely used ON/OFF observation method [22]. We define the different OFF regions according to Table 3. Given the stochastic nature of the emission of astrophysical gamma-ray photons and the detection of these by CTA, we create 50 simulated observations for the SHMF $\alpha=1.9 \mathrm{DM}$ model case and average the results in order to obtain statistically meaningful results.

As a first order approximation, we assume Perseus cluster to be a point-like source. No signal is found in these simulations, thus we compute the $95 \%$ confidence level (C.L.) upper limits to the gamma-ray flux. Then, we proceed and compute 95\% C.L. upper limits to the DM annihilation

1https://docs.gammapy.org/0.18.2/index.html

${ }^{2}$ http://cta.irap.omp.eu/ctools/ 


\begin{tabular}{|c|c|}
\hline $\mathrm{N}_{\text {obs }}$ & 50 \\
\hline $\mathrm{T}_{\text {obs }}[\mathrm{h}]$ & 300 \\
\hline Obs. strategy & 1 ON / 3 OFF \\
\hline Pointing $(l, b)[\mathrm{deg}]$ & $(150.57,-13.26)$ \\
\hline Offset $[\mathrm{deg}]$ & 1.0 \\
\hline On Region $[\mathrm{deg}]$ & 1.0 \\
\hline Energy range $[\mathrm{TeV}]$ & $0.03-100$ \\
\hline
\end{tabular}

Table 3: Observation parameters set-up for the Perseus DM-search campaign with the ON/OFF method.

cross section (Eq. 2) and decay lifetime (Eq. 3). The preliminary results are shown in Figure 3 for the $b \bar{b}, \tau^{+} \tau^{-}$and $W^{+} W^{-}$channels.

From the left panel of Figure 3 we can see that our constraints to DM annihilation are, at best, around two orders of magnitude above the thermal relic cross section, even with the inclusion of halo substructure. Yet, for decay DM, the results of the right panel show that CTA will be able to improve the limits from [23] by up to two orders of magnitude in the lowest considered mass range.
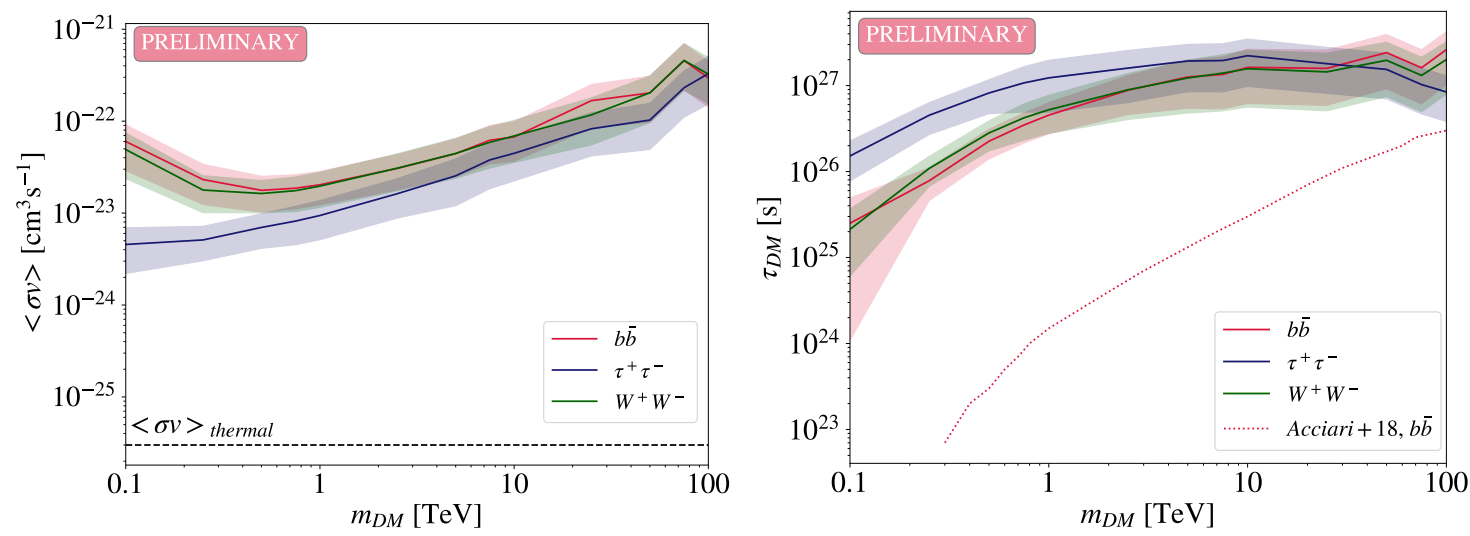

Figure 3: $95 \%$ C.L. upper limits on the DM parameter space. Left panel: Averaged annihilation cross section versus DM mass. The thermal relic cross section is shown as a black dashed line. Right panel: DM particle lifetime versus DM mass. For comparison, we show in red dotted line the constraints obtained from $202 \mathrm{~h}$ observation of Perseus for the $b \bar{b}$ channel with the MAGIC telescopes [23].

In the future, our analysis will necessarily consider the three known gamma-ray sources in Perseus, as well as the extension of the involved gamma-ray signals. An extended analysis, i.e. taking into account the extension of the cluster, is expected to worsen the obtained DM constraints. We will also include systematic uncertainties. An alternative analysis approach will also be performed via a template analysis fitting, as done for CTA in [24]. The result of this analysis yield the first DM annihilation constraints for Perseus from any IACT, that are complementary to the ones obtained by the Fermi-LAT Collaboration [25-27].

\section{References}

[1] G.M. Voit, Tracing cosmic evolution with clusters of galaxies, Rev. Mod. Phys. 77 (2005) 207. 
[2] R.J. van Weeren, F. de Gasperin, H. Akamatsu, M. Brüggen, L. Feretti, H. Kang et al., Diffuse Radio Emission from Galaxy Clusters, Space Sci. Rev. 215 (2019) 16 [1901.04496].

[3] A. Pinzke and C. Pfrommer, Simulating the $\gamma$-ray emission from galaxy clusters: a universal cosmic ray spectrum and spatial distribution, 409 (2010) 449 [1001.5023].

[4] M. Cirelli, G. Corcella, A. Hektor, G. Hutsi, M. Kadastik, P. Panci et al., PPPC 4 DM ID: A Poor Particle Physicist Cookbook for Dark Matter Indirect Detection, JCAP 03 (2011) 051 [1012 . 4515].

[5] Fermi-LAT collaboration, Search for cosmic-ray induced gamma-ray emission in Galaxy Clusters, Astrophys. J. 787 (2014) 18 [1308 . 5654].

[6] T.E. Jeltema, J. Kehayias and S. Profumo, Gamma Rays from Clusters and Groups of Galaxies: Cosmic Rays versus Dark Matter, Phys. Rev. D 80 (2009) 023005 [0812 . 0597].

[7] M.A. Sánchez-Conde, M. Cannoni, F. Zandanel, M.E. Gómez and F. Prada, Dark matter searches with Cherenkov telescopes: nearby dwarf galaxies or local galaxy clusters?, 2011 (2011) 011 [1104.3530].

[8] CTA Consortium collaboration, B.S. Acharya et al., Science with the Cherenkov Telescope Array, WSP (11, 2018), 10.1142/10986, [1709.07997].

[9] J. Aleksić, L.A. Antonelli, P. Antoranz, M. Backes, C. Baixeras, S. Balestra et al., MAGIC Gamma-ray Telescope Observation of the Perseus Cluster of Galaxies: Implications for Cosmic Rays, Dark Matter, and NGC 1275, 710 (2010) 634 [0909. 3267].

[10] G. Bertone, D. Hooper and J. Silk, Particle dark matter: Evidence, candidates and constraints, Phys. Rept. 405 (2005) 279 [hep-ph/0404175].

[11] Нгтомі collaboration, Atmospheric gas dynamics in the Perseus cluster observed with Hitomi, Publ. Astron. Soc. Jap. 70 (2018) 9 [1711.00240].

[12] J.F. Navarro, C.S. Frenk and S.D.M. White, The Structure of cold dark matter halos, Astrophys. J. 462 (1996) 563 [astro-ph/9508025].

[13] J.F. Navarro, C.S. Frenk and S.D.M. White, A Universal density profile from hierarchical clustering, Astrophys. J. 490 (1997) 493 [astro-ph/9611107].

[14] M.A. Sánchez-Conde and F. Prada, The flattening of the concentration-mass relation towards low halo masses and its implications for the annihilation signal boost, Mon. Not. Roy. Astron. Soc. 442 (2014) 2271 [1312 . 1729].

[15] J. Diemand, M. Kuhlen, P. Madau, M. Zemp, B. Moore, D. Potter et al., Clumps and streams in the local dark matter distribution, Nature 454 (2008) 735 [0805 . 1244].

[16] V. Springel, J. Wang, M. Vogelsberger, A. Ludlow, A. Jenkins, A. Helmi et al., The Aquarius Project: the subhalos of galactic halos, Mon. Not. Roy. Astron. Soc. 391 (2008) 1685 [0809.0898].

[17] A. Moliné, M.A. Sánchez-Conde, S. Palomares-Ruiz and F. Prada, Characterization of subhalo structural properties and implications for dark matter annihilation signals, Mon. Not. Roy. Astron. Soc. 466 (2017) 4974 [1603.04057].

[18] A. Charbonnier, C. Combet and D. Maurin, CLUMPY: a code for gamma-ray signals from dark matter structures, Comput. Phys. Commun. 183 (2012) 656 [1201.4728].

[19] V. Bonnivard, M. Hütten, E. Nezri, A. Charbonnier, C. Combet and D. Maurin, CLUMPY : Jeans analysis, $\gamma$-ray and $v$ fluxes from dark matter (sub-)structures, Comput. Phys. Commun. 200 (2016) 336 [1506.07628].

[20] M. Hütten, C. Combet and D. Maurin, CLUMPY v3: $\gamma$-ray and v signals from dark matter at all scales, Comput. Phys. Commun. 235 (2019) 336 [1806 . 08639]. 
[21] Fermi-LAT collaboration, Sensitivity Projections for Dark Matter Searches with the Fermi Large Area Telescope, Phys. Rept. 636 (2016) 1 [1605.02016].

[22] T.P. Li and Y.Q. Ma, Analysis methods for results in gamma-ray astronomy., 272 (1983) 317.

[23] MAGIC collaboration, Constraining Dark Matter lifetime with a deep gamma-ray survey of the Perseus Galaxy Cluster with MAGIC, Phys. Dark Univ. 22 (2018) 38 [1806.11063].

[24] CTA collaboration, Sensitivity of the Cherenkov Telescope Array to a dark matter signal from the Galactic centre, JCAP 01 (2021) 057 [2007.16129].

[25] M. Ackermann, M. Ajello, A. Allafort, L. Baldini, J. Ballet, G. Barbiellini et al., Constraints on dark matter annihilation in clusters of galaxies with the Fermi large area telescope, 2010 (2010) 025 [1002 .2239].

[26] FERMI-LAT collaboration, Galaxy Clusters with the Fermi-LAT: Status and Implications for Cosmic Rays and Dark Matter Physics, 1502.02653.

[27] C. Thorpe-Morgan, D. Malyshev, C.-A. Stegen, A. Santangelo and J. Jochum, Annihilating dark matter search with 12 yr of Fermi LAT data in nearby galaxy clusters, Mon. Not. Roy. Astron. Soc. 502 (2021) 4039 [2010 . 11006].

\section{The Cherenkov Telescope Array Consortium July 2021 Authors}

H. Abdalla ${ }^{1}$, H. Abe ${ }^{2}$, S. Abe ${ }^{2}$, A. Abusleme ${ }^{3}$, F. Acero ${ }^{4}$, A. Acharyya ${ }^{5}$, V. Acín Portella ${ }^{6}$, K. Ackley ${ }^{7}$, R. Adam ${ }^{8}$, C. Adams ${ }^{9}$, S.S. Adhikari ${ }^{10}$, I. Aguado-Ruesga ${ }^{11}$, I. Agudo ${ }^{12}$, R. Aguilera ${ }^{13}$, A. Aguirre-Santaella ${ }^{14}$, F. Aharonian ${ }^{15}$, A. Alberdi ${ }^{12}$, R. Alfaro ${ }^{16}$, J. Alfaro ${ }^{3}$, C. Alispach ${ }^{17}$, R. Aloisio ${ }^{18}$, R. Alves Batista ${ }^{19}$, J.-P. Amans ${ }^{20}$, L. Amati ${ }^{21}$, E. Amato ${ }^{22}$, L. Ambrogi ${ }^{18}$, G. Ambrosi $^{23}$, M. Ambrosio ${ }^{24}$, R. Ammendola ${ }^{25}$, J. Anderson ${ }^{26}$, M. Anduze $^{8}$, E.O. Angüner ${ }^{27}$, L.A. Antonelli ${ }^{28}$, V. Antonuccio ${ }^{29}$, P. Antoranz ${ }^{30}$, R. Anutarawiramkul ${ }^{31}$, J. Aragunde Gutierrez ${ }^{32}$, C. Aramo ${ }^{24}$, A. Araudo ${ }^{33,34}$, M. Araya ${ }^{35}$, A. Arbet-Engels ${ }^{36}$, C. Arcaro ${ }^{1}$, V. Arendt ${ }^{37}$, C. Armand ${ }^{38}$, T. Armstrong ${ }^{27}$,

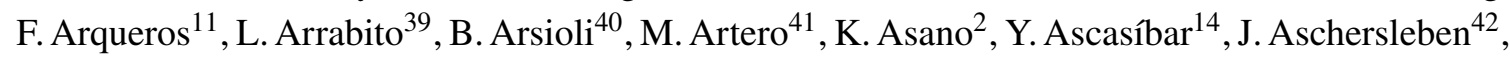
M. Ashley ${ }^{43}$, P. Attinà ${ }^{44}$, P. Aubert ${ }^{45}$, C. B. Singh ${ }^{19}$, D. Baack ${ }^{46}$, A. Babic ${ }^{47}$, M. Backes ${ }^{48}$, V. Baena ${ }^{13}$, S. Bajtlik ${ }^{49}$, A. Baktash ${ }^{50}$, C. Balazs ${ }^{7}$, M. Balbo ${ }^{38}$, O. Ballester ${ }^{41}$, J. Ballet ${ }^{4}$, B. Balmaverde ${ }^{44}$, A. Bamba ${ }^{51}$, R. Bandiera ${ }^{22}$, A. Baquero Larriva ${ }^{11}$, P. Barai ${ }^{19}$, C. Barbier ${ }^{45}$, V. Barbosa Martins ${ }^{52}$, M. Barcelo ${ }^{53}$, M. Barkov ${ }^{54}$, M. Barnard ${ }^{1}$, L. Baroncelli ${ }^{21}$, U. Barres de Almeida ${ }^{40}$, J.A. Barrio ${ }^{11}$, D. Bastieri ${ }^{55}$, P.I. Batista ${ }^{52}$, I. Batkovic ${ }^{55}$, C. Bauer ${ }^{53}$, R. Bautista-González ${ }^{56}$, J. Baxter ${ }^{2}$, U. Becciani ${ }^{29}$, J. Becerra González ${ }^{32}$, Y. Becherini ${ }^{57}$, G. Beck ${ }^{58}$, J. Becker Tjus ${ }^{59}$, W. Bednarek ${ }^{60}$, A. Belfiore ${ }^{61}$, L. Bellizzi ${ }^{62}$, R. Belmont ${ }^{4}$, W. Benbow ${ }^{63}$, D. Berge ${ }^{52}$, E. Bernardini ${ }^{52}$, M.I. Bernardos ${ }^{55}$, K. Bernlöhr $^{53}$, A. Berti ${ }^{64}$, M. Berton ${ }^{65}$, B. Bertucci ${ }^{23}$, V. Beshley ${ }^{66}$, N. Bhatt ${ }^{67}$, S. Bhattacharyya ${ }^{67}$, W. Bhattacharyya ${ }^{52}$, S. Bhattacharyya ${ }^{68}$, B. Bi ${ }^{69}$, G. Bicknell ${ }^{70}$, N. Biederbeck ${ }^{46}$, C. Bigongiari ${ }^{28}$, A. Biland ${ }^{36}$, R. Bird ${ }^{71}$,

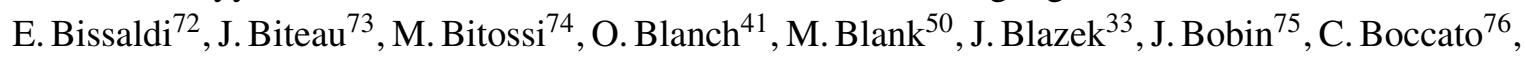
F. Bocchino ${ }^{77}$, C. Boehm ${ }^{78}$, M. Bohacova ${ }^{33}$, C. Boisson ${ }^{20}$, J. Boix ${ }^{41}$, J.-P. Bolle ${ }^{52}$, J. Bolmont ${ }^{79}$, G. Bonanno ${ }^{29}$, C. Bonavolontà ${ }^{24}$, L. Bonneau Arbeletche ${ }^{80}$, G. Bonnoli $^{12}$, P. Bordas ${ }^{81}$, J. Borkowski ${ }^{49}$, S. Bórquez ${ }^{35}$, R. Bose ${ }^{82}$, D. Bose ${ }^{83}$, Z. Bosnjak ${ }^{47}$, E. Bottacini ${ }^{55}$, M. Böttcher ${ }^{1}$, M.T. Botticella ${ }^{84}$, C. Boutonnet ${ }^{85}$, F. Bouyjou ${ }^{75}$, V. Bozhilov ${ }^{86}$, E. Bozzo ${ }^{38}$, L. Brahimi ${ }^{39}$, C. Braiding ${ }^{43}$, S. BrauNogué $^{87}$, S. Breen ${ }^{78}$, J. Bregeon ${ }^{39}$, M. Breuhaus ${ }^{53}$, A. Brill ${ }^{9}$, W. Brisken ${ }^{88}$, E. Brocato ${ }^{28}$, A.M. Brown ${ }^{5}$, K. Brügge ${ }^{46}$, P. Brun ${ }^{89}$, P. Brun ${ }^{39}$, F. Brun ${ }^{89}$, L. Brunetti ${ }^{45}$, G. Brunetti ${ }^{90}$, P. Bruno ${ }^{29}$, A. Bruno ${ }^{91}$, A. Bruzzese ${ }^{6}$, N. Bucciantini ${ }^{22}$, J. Buckley ${ }^{82}$, R. Bühler ${ }^{52}$, A. Bulgarelli ${ }^{21}$, T. Bulik ${ }^{92}$, M. Bünning ${ }^{52}$, M. Bunse ${ }^{46}$, M. Burton ${ }^{93}$, A. Burtovoi ${ }^{76}$, M. Buscemi ${ }^{94}$, S. Buschjäger ${ }^{46}$, G. Busetto ${ }^{55}$, J. Buss ${ }^{46}$, 
K. Byrum ${ }^{26}$, A. Caccianiga ${ }^{95}$, F. Cadoux ${ }^{17}$, A. Calanducci ${ }^{29}$, C. Calderón ${ }^{3}$, J. Calvo Tovar ${ }^{32}$, R. Cameron ${ }^{96}$, P. Campaña ${ }^{35}$, R. Canestrari ${ }^{91}$, F. Cangemi ${ }^{79}$, B. Cantlay ${ }^{31}$, M. Capalbi ${ }^{91}$, M. Capasso ${ }^{9}$, M. Cappi $^{21}$, A. Caproni ${ }^{97}$, R. Capuzzo-Dolcetta ${ }^{28}$, P. Caraveo ${ }^{61}$, V. Cárdenas ${ }^{98}$, L. Cardiel ${ }^{41}$, M. Cardillo ${ }^{99}$, C. Carlile ${ }^{100}$, S. Caroff ${ }^{45}$, R. Carosi ${ }^{74}$, A. Carosi ${ }^{17}$, E. Carquín ${ }^{35}$, M. Carrère ${ }^{39}$, J.-M. Casandjian ${ }^{4}$, S. Casanova ${ }^{101,53}$, E. Cascone ${ }^{84}$, F. Cassol ${ }^{27}$, A.J. Castro-Tirado ${ }^{12}$, F. Catalani ${ }^{102}$, O. Catalano ${ }^{91}$, D. Cauz ${ }^{103}$, A. Ceccanti ${ }^{64}$, C. Celestino Silva ${ }^{80}$, S. Celli ${ }^{18}$, K. Cerny ${ }^{104}$, M. Cerruti ${ }^{85}$, E. Chabanne ${ }^{45}$, P. Chadwick ${ }^{5}$, Y. Chai ${ }^{105}$, P. Chambery ${ }^{106}$, C. Champion ${ }^{85}$, S. Chandra ${ }^{1}$, S. Chaty ${ }^{4}$, A. Chen ${ }^{58}$, K. Cheng ${ }^{2}$, M. Chernyakova ${ }^{107}$, G. Chiaro ${ }^{61}$, A. Chiavassa ${ }^{64,108}$, M. Chikawa ${ }^{2}$, V.R. Chitnis ${ }^{109}$, J. Chudoba ${ }^{33}$, L. Chytka ${ }^{104}$, S. Cikota ${ }^{47}$, A. Circiello ${ }^{24,110}$, P. Clark ${ }^{5}$, M. Çolak ${ }^{41}$, E. Colombo ${ }^{32}$, J. Colome ${ }^{13}$, S. Colonges ${ }^{85}$, A. Comastri ${ }^{21}$, A. Compagnino ${ }^{91}$, V. Conforti ${ }^{21}$, E. Congiu ${ }^{95}$, R. Coniglione ${ }^{94}$, J. Conrad ${ }^{111}$, F. Conte ${ }^{53}$, J.L. Contreras ${ }^{11}$, P. Coppi ${ }^{112}$, R. Cornat ${ }^{8}$, J. Coronado-Blazquez ${ }^{14}$, J. Cortina ${ }^{113}$, A. Costa $^{29}$, H. Costantini ${ }^{27}$, G. Cotter ${ }^{114}$, B. Courty ${ }^{85}$, S. Covino ${ }^{95}$, S. Crestan ${ }^{61}$, P. Cristofari ${ }^{20}$, R. Crocker ${ }^{70}$, J. Croston ${ }^{115}$, K. Cubuk ${ }^{93}$, O. Cuevas ${ }^{98}$, X. Cui ${ }^{2}$, G. Cusumano ${ }^{91}$, S. Cutini ${ }^{23}$, A. D’Aì ${ }^{91}$, G. D’Amico ${ }^{116}$, F. D'Ammando ${ }^{90}$, P. D’Avanzo ${ }^{95}$, P. Da Vela ${ }^{74}$, M. Dadina ${ }^{21}$, S. Dai ${ }^{117}$, M. Dalchenko ${ }^{17}$, M. Dall' Ora ${ }^{84}$, M.K. Daniel ${ }^{63}$, J. Dauguet ${ }^{85}$, I. Davids ${ }^{48}$, J. Davies ${ }^{114}$, B. Dawson ${ }^{118}$, A. De Angelis ${ }^{55}$, A.E. de Araújo Carvalho ${ }^{40}$, M. de Bony de Lavergne ${ }^{45}$, V. De Caprio $^{84}$, G. De Cesare ${ }^{21}$, F. De Frondat ${ }^{20}$, E.M. de Gouveia Dal Pino ${ }^{19}$, I. de la Calle ${ }^{11}$, B. De Lotto ${ }^{103}$, A. De Luca ${ }^{61}$, D. De Martino ${ }^{84}$, R.M. de Menezes ${ }^{19}$, M. de Naurois ${ }^{8}$, E. de Oña Wilhelmi ${ }^{13}$, F. De Palma ${ }^{64}$, F. De Persio ${ }^{119}$, N. de Simone ${ }^{52}$, V. de Souza ${ }^{80}$, M. Del Santo $^{91}$, M.V. del Valle ${ }^{19}$, E. Delagnes ${ }^{75}$, G. Deleglise ${ }^{45}$, M. Delfino Reznicek ${ }^{6}$, C. Delgado ${ }^{113}$, A.G. Delgado Giler ${ }^{80}$, J. Delgado Mengual ${ }^{6}$, R. Della $\mathrm{Ceca}^{95}$, M. Della Valle ${ }^{84}$, D. della Volpe ${ }^{17}$, D. Depaoli ${ }^{64,108}$, D. Depouez ${ }^{27}$, J. Devin ${ }^{85}$, T. Di Girolamo ${ }^{24,110}$, C. Di Giulio ${ }^{25}$, A. Di Piano ${ }^{21}$, F. Di Pierro $^{64}$, L. Di Venere ${ }^{120}$, C. Díaz ${ }^{113}$, C. Díaz-Bahamondes ${ }^{3}$, C. Dib ${ }^{35}$, S. Diebold ${ }^{69}$, S. Digel ${ }^{96}$, R. Dima ${ }^{55}$, A. Djannati-Atai ${ }^{85}$, J. Djuvsland ${ }^{116}$, A. Dmytriiev ${ }^{20}$, K. Docher ${ }^{9}$, A. Domínguez ${ }^{11}$, D. Dominis Prester ${ }^{121}$, A. Donath ${ }^{53}$, A. Donini ${ }^{41}$, D. Dorner ${ }^{122}$, M. Doro ${ }^{55}$, R.d.C. dos Anjos ${ }^{123}$, J.-L. Dournaux ${ }^{20}$, T. Downes ${ }^{107}$, G. Drake ${ }^{26}$, H. Drass $^{3}$, D. Dravins ${ }^{100}$, C. Duangchan ${ }^{31}$, A. Duara ${ }^{124}$, G. Dubus ${ }^{125}$, L. Ducci ${ }^{69}$, C. Duffy ${ }^{124}$, D. Dumora ${ }^{106}$, K. Dundas Morå ${ }^{111}$, A. Durkalec ${ }^{126}$, V.V. Dwarkadas ${ }^{127}$, J. Ebr ${ }^{33}$, C. Eckner ${ }^{45}$, J. Eder ${ }^{105}$, A. Ederoclite ${ }^{19}$, E. Edy ${ }^{8}$, K. Egberts ${ }^{128}$, S. Einecke ${ }^{118}$, J. Eisch ${ }^{129}$, C. Eleftheriadis ${ }^{130}$, D. Elsässer ${ }^{46}$, G. Emery ${ }^{17}$, D. Emmanoulopoulos ${ }^{115}$, J.-P. Ernenwein ${ }^{27}$, M.Errando ${ }^{82}$, P. Escarate ${ }^{35}$, J. Escudero ${ }^{12}$, C. Espinoza ${ }^{3}$, S. Ettori ${ }^{21}$, A. Eungwanichayapant ${ }^{31}$, P. Evans ${ }^{124}$, C. Evoli ${ }^{18}$, M. Fairbairn ${ }^{131}$, D. Falceta-Goncalves ${ }^{132}$, A. Falcone ${ }^{133}$, V. Fallah Ramazani ${ }^{65}$, R. Falomo ${ }^{76}$, K. Farakos ${ }^{134}$, G. Fasola ${ }^{20}$, A. Fattorini ${ }^{46}$, Y. Favre ${ }^{17}$, R. Fedora ${ }^{135}$, E. Fedorova ${ }^{136}$, S. Fegan ${ }^{8}$, K. Feijen ${ }^{118}$, Q. Feng ${ }^{9}$, G. Ferrand ${ }^{54}$, G. Ferrara ${ }^{94}$, O. Ferreira ${ }^{8}$, M. Fesquet ${ }^{75}$, E. Fiandrini $^{23}$, A. Fiasson ${ }^{45}$, M. Filipovic ${ }^{117}$, D. Fink ${ }^{105}$, J.P. Finley ${ }^{137}$, V. Fioretti ${ }^{21}$, D.F.G. Fiorillo ${ }^{24,110}$, M. Fiorini ${ }^{61}$, S. Flis ${ }^{52}$, H. Flores ${ }^{20}$, L. Foffano ${ }^{17}$, C. Föhr ${ }^{53}$, M.V. Fonseca ${ }^{11}$, L. Font ${ }^{138}$, G. Fontaine ${ }^{8}$, O. Fornieri $^{52}$, P. Fortin ${ }^{63}$, L. Fortson ${ }^{88}$, N. Fouque ${ }^{45}$, A. Fournier ${ }^{106}$, B. Fraga ${ }^{40}$, A. Franceschini ${ }^{76}$, F.J. Franco ${ }^{30}$, A. Franco Ordovas ${ }^{32}$, L. Freixas Coromina ${ }^{113}$, L. Fresnillo ${ }^{30}$, C. Fruck ${ }^{105}$, D. Fugazza ${ }^{95}$, Y. Fujikawa ${ }^{139}$, Y. Fujita ${ }^{2}$, S. Fukami ${ }^{2}$, Y. Fukazawa ${ }^{140}$, Y. Fukui ${ }^{141}$, D. Fulla ${ }^{52}$, S. Funk ${ }^{142}$, A. Furniss ${ }^{143}$, O. Gabella ${ }^{39}$, S. Gabici ${ }^{85}$, D. Gaggero ${ }^{14}$, G. Galanti ${ }^{61}$, G. Galaz $^{3}$, P. Galdemard ${ }^{144}$, Y. Gallant ${ }^{39}$, D. Galloway ${ }^{7}$, S. Gallozzi ${ }^{28}$, V. Gammaldi ${ }^{14}$, R. Garcia ${ }^{41}$, E. Garcia ${ }^{45}$, E. García ${ }^{13}$, R. Garcia López ${ }^{32}$, M. Garczarczyk ${ }^{52}$, F. Gargano ${ }^{120}$, C. Gargano ${ }^{91}$, S. Garozzo ${ }^{29}$, D. Gascon ${ }^{81}$, T. Gasparetto ${ }^{145}$, D. Gasparrini ${ }^{25}$, H. Gasparyan ${ }^{52}$, M. Gaug ${ }^{138}$, N. Geffroy ${ }^{45}$, A. Gent ${ }^{146}$, S. Germani ${ }^{76}$, L. Gesa ${ }^{13}$, A. Ghalumyan ${ }^{147}$, A. Ghedina ${ }^{148}$, G. Ghirlanda ${ }^{95}$, F. Gianotti ${ }^{21}$, S. Giarrusso ${ }^{91}$, M. Giarrusso ${ }^{94}$, 
G. Giavitto $^{52}$, B. Giebels ${ }^{8}$, N. Giglietto ${ }^{72}$, V. Gika ${ }^{134}$, F. Gillardo ${ }^{45}$, R. Gimenes ${ }^{19}$, F. Giordano ${ }^{149}$, G. Giovannini ${ }^{90}$, E. Giro ${ }^{76}$, M. Giroletti ${ }^{90}$, A. Giuliani ${ }^{61}$, L. Giunti ${ }^{85}$, M. Gjaja ${ }^{9}$, J.-F. Glicenstein ${ }^{89}$, P. Gliwny ${ }^{60}$, N. Godinovic ${ }^{150}$, H. Göksu ${ }^{53}$, P. Goldoni ${ }^{85}$, J.L. Gómez ${ }^{12}$, G. Gómez-Vargas ${ }^{3}$, M.M. González ${ }^{16}$, J.M. González ${ }^{151}$, K.S. Gothe ${ }^{109}$, D. Götz ${ }^{4}$, J. Goulart Coelho ${ }^{123}$, K. Gourgouliatos ${ }^{5}$, T. Grabarczyk ${ }^{152}$, R. Graciani ${ }^{81}$, P. Grandi ${ }^{21}$, G. Grasseau $^{8}$, D. Grasso ${ }^{74}$, A.J. Green ${ }^{78}$, D. Green ${ }^{105}$, J. Green ${ }^{28}$, T. Greenshaw ${ }^{153}$, I. Grenier ${ }^{4}$, P. Grespan ${ }^{55}$, A. Grillo ${ }^{29}$, M.-H. Grondin ${ }^{106}$, J. Grube ${ }^{131}$, V. Guarino ${ }^{26}$, B. Guest ${ }^{37}$, O. Gueta ${ }^{52}$, M. Gündüz ${ }^{59}$, S. Gunji ${ }^{154}$, A. Gusdorf ${ }^{20}$, G. Gyuk ${ }^{155}$, J. Hackfeld ${ }^{59}$, D. Hadasch ${ }^{2}$, J. Haga ${ }^{139}$, L. Hagge ${ }^{52}$, A. Hahn ${ }^{105}$, J.E. Hajlaoui ${ }^{85}$, H. Hakobyan ${ }^{35}$,

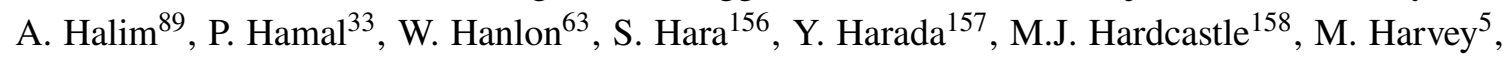
K. Hashiyama ${ }^{2}$, T. Hassan Collado ${ }^{113}$, T. Haubold ${ }^{105}$, A. Haupt ${ }^{52}$, U.A. Hautmann ${ }^{159}$, M. Havelka ${ }^{33}$, K. Hayashi ${ }^{141}$, K. Hayashi ${ }^{160}$, M. Hayashida ${ }^{161}$, H. He ${ }^{54}$, L. Heckmann ${ }^{105}$, M. Heller ${ }^{17}$, J.C. Helo ${ }^{35}$, F. Henault ${ }^{125}$, G. Henri ${ }^{125}$, G. Hermann ${ }^{53}$, R. Hermel ${ }^{45}$, S. Hernández Cadena ${ }^{16}$, J. Herrera Llorente $^{32}$, A. Herrero ${ }^{32}$, O. Hervet ${ }^{143}$, J. Hinton ${ }^{53}$, A. Hiramatsu ${ }^{157}$, N. Hiroshima ${ }^{54}$, K. Hirotani ${ }^{2}$, B. Hnatyk ${ }^{136}$, R. Hnatyk ${ }^{136}$, J.K. Hoang ${ }^{11}$, D. Hoffmann ${ }^{27}$, W. Hofmann ${ }^{53}$, C. Hoischen ${ }^{128}$, J. Holder ${ }^{162}$, M. Holler ${ }^{163}$, B. Hona ${ }^{164}$, D. Horan ${ }^{8}$, J. Hörandel ${ }^{165}$, D. Horns ${ }^{50}$, P. Horvath ${ }^{104}$,

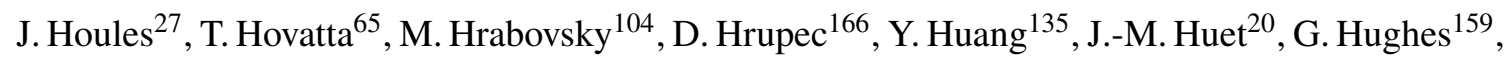

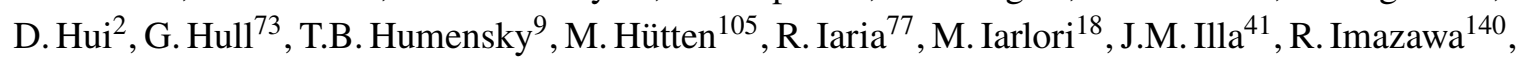
D. Impiombato ${ }^{91}$, T. Inada $^{2}$, F. Incardona ${ }^{29}$, A. Ingallinera ${ }^{29}$, Y. Inome ${ }^{2}$, S. Inoue ${ }^{54}$, T. Inoue ${ }^{141}$, Y. Inoue ${ }^{167}$, A. Insolia ${ }^{120,94}$, F. Iocco ${ }^{24,110}$, K. Ioka ${ }^{168}$, M. Ionica ${ }^{23}$, M. Iori ${ }^{119}$, S. Iovenitti ${ }^{95}$, A. Iriarte ${ }^{16}$, K. Ishio ${ }^{105}$, W. Ishizaki ${ }^{168}$, Y. Iwamura ${ }^{2}$, C. Jablonski ${ }^{105}$, J. Jacquemier ${ }^{45}$, M. Jacquemont ${ }^{45}$, M. Jamrozy ${ }^{169}$, P. Janecek ${ }^{33}$, F. Jankowsky ${ }^{170}$, A. Jardin-Blicq ${ }^{31}$, C. Jarnot ${ }^{87}$, P. Jean ${ }^{87}$, I. Jiménez Martínez $^{113}$, W. Jin ${ }^{171}$, L. Jocou ${ }^{125}$, N. Jordana ${ }^{172}$, M. Josselin ${ }^{73}$, L. Jouvin ${ }^{41}$, I. Jung-Richardt ${ }^{142}$, F.J.P.A. Junqueira ${ }^{19}$, C. Juramy-Gilles ${ }^{79}$, J. Jurysek ${ }^{38}$, P. Kaaret ${ }^{173}$, L.H.S. Kadowaki ${ }^{19}$, M. Kagaya ${ }^{2}$, O. Kalekin ${ }^{142}$, R. Kankanyan ${ }^{53}$, D. Kantzas ${ }^{174}$, V. Karas ${ }^{34}$, A. Karastergiou ${ }^{114}$, S. Karkar ${ }^{79}$, E. Kasai ${ }^{48}$, J. Kasperek ${ }^{175}$, H. Katagiri ${ }^{176}$, J. Kataoka ${ }^{177}$, K. Katarzyński ${ }^{178}$, S. Katsuda ${ }^{179}$, U. Katz ${ }^{142}$, N. Kawanaka ${ }^{180}$, D. Kazanas ${ }^{130}$, D. Kerszberg ${ }^{41}$, B. Khélifi ${ }^{85}$, M.C. Kherlakian ${ }^{52}$, T.P. Kian ${ }^{181}$, D.B. Kieda ${ }^{164}$, T. Kihm ${ }^{53}$, S. Kim ${ }^{3}$, S. Kimeswenger ${ }^{163}$, S. Kisaka ${ }^{140}$, R. Kissmann ${ }^{163}$, R. Kleijwegt ${ }^{135}$, T. Kleiner ${ }^{52}$, G. Kluge ${ }^{10}$, W. Kluźniak ${ }^{49}$, J. Knapp ${ }^{52}$, J. Knödlseder ${ }^{87}$, A. Kobakhidze ${ }^{78}$, Y. Kobayashi ${ }^{2}$, B. Koch ${ }^{3}$, J. Kocot ${ }^{152}$, K. Kohri ${ }^{182}$, K. Kokkotas ${ }^{69}$, N. Kominn', A. Kong ${ }^{2}$, K. Kosack ${ }^{4}$, G. Kowal ${ }^{132}$, F. Krack ${ }^{52}$, M. Krause ${ }^{52}$, F. Krennrich ${ }^{129}$, M. Krumholz ${ }^{70}$, H. Kubo ${ }^{180}$, V. Kudryavtsev ${ }^{183}$, S. Kunwar ${ }^{53}$, Y. Kuroda ${ }^{139}$, J. Kushida ${ }^{157}$, P. Kushwaha ${ }^{19}$, A. La Barbera ${ }^{91}$, N. La Palombara ${ }^{61}$, V. La Parola ${ }^{91}$, G. La Rosa ${ }^{91}$, R. Lahmann ${ }^{142}$, G. Lamanna ${ }^{45}$, A. Lamastra ${ }^{28}$, M. Landoni ${ }^{95}$, D. Landriu ${ }^{4}$, R.G. Lang ${ }^{80}$, J. Lapington ${ }^{124}$, P. Laporte ${ }^{20}$, P. Lason ${ }^{152}$, J. Lasuik ${ }^{37}$, J. Lazendic-Galloway ${ }^{7}$, T. Le Flour ${ }^{45}$, P. Le Sidaner ${ }^{20}$, S. Leach ${ }^{124}$, A. Leckngam ${ }^{31}$, S.-H. Lee ${ }^{180}$, W.H. Lee ${ }^{16}$, S. Lee ${ }^{118}$, M.A. Leigui de Oliveira ${ }^{184}$, A. Lemière ${ }^{85}$, M. Lemoine-Goumard ${ }^{106}$, J.-P. Lenain ${ }^{79}$, F. Leone ${ }^{94,185}$, V. Leray ${ }^{8}$, G. Leto $^{29}$, F. Leuschner ${ }^{69}$, C. Levy ${ }^{79,20}$, R. Lindemann ${ }^{52}$, E. Lindfors ${ }^{65}$, L. Linhoff ${ }^{46}$, I. Liodakis ${ }^{65}$, A. Lipniacka ${ }^{116}$, S. Lloyd ${ }^{5}$, M. Lobo ${ }^{113}$, T. Lohse ${ }^{186}$, S. Lombardi ${ }^{28}$, F. Longo ${ }^{145}$, A. Lopez ${ }^{32}$, M. López ${ }^{11}$, R. López-Coto ${ }^{55}$, S. Loporchio ${ }^{149}$, F. Louis $^{75}$, M. Louys ${ }^{20}$, F. Lucarelli ${ }^{28}$, D. Lucchesi ${ }^{55}$, H. Ludwig Boudi ${ }^{39}$, P.L. Luque-Escamilla ${ }^{56}$, E. Lyard $^{38}$, M.C. Maccarone ${ }^{91}$, T. Maccarone ${ }^{187}$, E. Mach ${ }^{101}$, A.J. Maciejewski ${ }^{188}$, J. Mackey ${ }^{15}$, G.M. Madejski ${ }^{96}$, P. Maeght ${ }^{39}$, C. Maggio ${ }^{138}$, G. Maier ${ }^{52}$, A. Majczyna ${ }^{126}$, P. Majumdar ${ }^{83,2}$, M. Makariev ${ }^{189}$, M. Mallamaci ${ }^{55}$, R. Malta Nunes de Almeida ${ }^{184}$, S. Maltezos ${ }^{134}$, D. Malyshev ${ }^{142}$, D. Malyshev ${ }^{69}$, D. Mandat ${ }^{33}$, G. Maneva ${ }^{189}$, M. Manganaro ${ }^{121}$, G. Manicò ${ }^{94}$, P. Manigot ${ }^{8}$, K. Mannheim ${ }^{122}$, 
N. Maragos ${ }^{134}$, D. Marano ${ }^{29}$, M. Marconi ${ }^{84}$, A. Marcowith ${ }^{39}$, M. Marculewicz ${ }^{190}$, B. Marčun ${ }^{68}$, J. Marín ${ }^{98}$, N. Marinello ${ }^{55}$, P. Marinos ${ }^{118}$, M. Mariotti $^{55}$, S. Markoff $^{174}$, P. Marquez $^{41}$, G. Marsella $^{94}$, J. Martí ${ }^{56}$, J.-M. Martin ${ }^{20}$, P. Martin ${ }^{87}$, O. Martinez ${ }^{30}$, M. Martínez $^{41}$, G. Martínez ${ }^{113}$, O. Martínez $^{41}$, H. Martínez-Huerta ${ }^{80}$, C. Marty ${ }^{87}$, R. Marx ${ }^{53}$, N. Masetti ${ }^{21,151}$, P. Massimino ${ }^{29}$, A. Mastichiadis ${ }^{191}$, H. Matsumoto ${ }^{167}$, N. Matthews ${ }^{164}$, G. Maurin ${ }^{45}$, W. Max-Moerbeck ${ }^{192}$, N. Maxted ${ }^{43}$, D. Mazin ${ }^{2,105}$, M.N. Mazziotta ${ }^{120}$, S.M. Mazzola ${ }^{77}$, J.D. Mbarubucyeye ${ }^{52}$, L. Mc Comb ${ }^{5}$, I. McHardy ${ }^{115}$, S. McKeague ${ }^{107}$, S. McMuldroch ${ }^{63}$, E. Medina ${ }^{64}$, D. Medina Miranda ${ }^{17}$, A. Melandri ${ }^{95}$, C. Melioli ${ }^{19}$, D. Melkumyan ${ }^{52}$, S. Menchiari ${ }^{62}$, S. Mender ${ }^{46}$, S. Mereghetti ${ }^{61}$, G. Merino Arévalo ${ }^{6}$, E. Mestre ${ }^{13}$, J.-L. Meunier ${ }^{79}$, T. Meures ${ }^{135}$, M. Meyer ${ }^{142}$, S. Micanovic ${ }^{121}$, M. Miceli ${ }^{77}$, M. Michailidis ${ }^{69}$, J. Michałowski ${ }^{101}$, T. Miener ${ }^{11}$, I. Mievre ${ }^{45}$, J. Miller ${ }^{35}$, I.A. Minaya ${ }^{153}$, T. Mineo ${ }^{91}$, M. Minev ${ }^{189}$, J.M. Miranda ${ }^{30}$, R. Mirzoyan ${ }^{105}$, A. Mitchell ${ }^{36}$, T. Mizuno ${ }^{193}$, B. Mode ${ }^{135}$, R. Moderski ${ }^{49}$, L. Mohrmann ${ }^{142}$, E. Molina ${ }^{81}$, E. Molinari ${ }^{148}$, T. Montaruli ${ }^{17}$, I. Monteiro ${ }^{45}$, C. Moore ${ }^{124}$, A. Moralejo ${ }^{41}$, D. MorcuendeParrilla $^{11}$, E. Moretti ${ }^{41}$, L. Morganti ${ }^{64}$, K. Mori ${ }^{194}$, P. Moriarty ${ }^{15}$, K. Morik ${ }^{46}$, G. Morlino ${ }^{22}$, P. Morris ${ }^{114}$, A. Morselli ${ }^{25}$, K. Mosshammer ${ }^{52}$, P. Moya ${ }^{192}$, R. Mukherjee ${ }^{9}$, J. Muller $^{8}$, C. Mundell ${ }^{172}$,

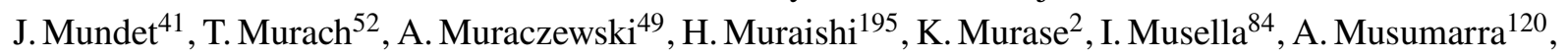
A. Nagai ${ }^{17}$, N. Nagar ${ }^{196}$, S. Nagataki ${ }^{54}$, T. Naito ${ }^{156}$, T. Nakamori ${ }^{154}$, K. Nakashima ${ }^{142}$, K. Nakayama ${ }^{51}$, N. Nakhjiri ${ }^{13}$, G. Naletto ${ }^{55}$, D. Naumann ${ }^{52}$, L. Nava ${ }^{95}$, R. Navarro ${ }^{174}$, M.A. Nawaz ${ }^{132}$, H. Ndiyavala ${ }^{1}$, D. Neise ${ }^{36}$, L. Nellen ${ }^{16}$, R. Nemmen ${ }^{19}$, M. Newbold ${ }^{164}$, N. Neyroud ${ }^{45}$, K. Ngernphat ${ }^{31}$, T. Nguyen Trung $^{73}$, L. Nicastro ${ }^{21}$, L. Nickel ${ }^{46}$, J. Niemiec ${ }^{101}$, D. Nieto ${ }^{11}$, M. Nievas ${ }^{32}$, C. Nigro ${ }^{41}$, M. Nikołajuk ${ }^{190}$, D. Ninci ${ }^{41}$, K. Nishijima ${ }^{157}$, K. Noda ${ }^{2}$, Y. Nogami ${ }^{176}$, S. Nolan ${ }^{5}$, R. Nomura ${ }^{2}$, R. Norris ${ }^{117}$, D. Nosek ${ }^{197}$, M. Nöthe ${ }^{46}$, B. Novosyadlyj ${ }^{198}$, V. Novotny ${ }^{197}$, S. Nozaki ${ }^{180}$, F. Nunio $^{144}$, P. O’ Brien ${ }^{124}$, K. Obara ${ }^{176}$, R. Oger ${ }^{85}$, Y. Ohira ${ }^{51}$, M. Ohishi ${ }^{2}$, S. Ohm ${ }^{52}$, Y. Ohtani ${ }^{2}$, T. Oka ${ }^{180}$, N. Okazaki ${ }^{2}$, A. Okumura ${ }^{139,199}$, J.-F. Olive ${ }^{87}$, C. Oliver $^{30}$, G. Olivera ${ }^{52}$, B. Olmi ${ }^{22}$, R.A. Ong ${ }^{71}$, M. Orienti ${ }^{90}$, R. Orito ${ }^{200}$, M. Orlandini ${ }^{21}$, S. Orlando ${ }^{77}$, E. Orlando ${ }^{145}$, J.P. Osborne ${ }^{124}$, M. Ostrowski ${ }^{169}$, N. Otte ${ }^{146}$, E. Ovcharov ${ }^{86}$, E. Owen ${ }^{2}$, I. Oya ${ }^{159}$, A. Ozieblo ${ }^{152}$, M. Padovani ${ }^{22}$, I. Pagano ${ }^{29}$, A. Pagliaro ${ }^{91}$, A. Paizis ${ }^{61}$, M. Palatiello ${ }^{145}$, M. Palatka ${ }^{33}$, E. Palazzi ${ }^{21}$, J.-L. Panazol ${ }^{45}$, D. Paneque ${ }^{105}$, B. Panes ${ }^{3}$, S. Panny ${ }^{163}$, F.R. Pantaleo ${ }^{72}$, M. Panter ${ }^{53}$, R. Paoletti ${ }^{62}$, M. Paolillo ${ }^{24,110}$, A. Papitto ${ }^{28}$, A. Paravac ${ }^{122}$, J.M. Paredes ${ }^{81}$, G. Pareschi ${ }^{95}$, N. Park ${ }^{127}$, N. Parmiggiani ${ }^{21}$, R.D. Parsons ${ }^{186}$, P. Paśko ${ }^{201}$, S. Patel ${ }^{52}$, B. Patricellii ${ }^{28}$, G. Pauletta ${ }^{103}$, L. Pavletić ${ }^{121}$, S. Pavy ${ }^{8}$, A. Pe'er ${ }^{105}$, M. Pech ${ }^{33}$, M. Pecimotika ${ }^{121}$, M.G. Pellegriti ${ }^{120}$, P. Peñil Del Campo ${ }^{11}$, M. Penno ${ }^{52}$, A. Pepato ${ }^{55}$, S. Perard ${ }^{106}$, C. Perennes ${ }^{55}$, G. Peres ${ }^{77}$, M. Peresano ${ }^{4}$, A. Pérez-Aguilera ${ }^{11}$, J. Pérez-Romero ${ }^{14}$, M.A. PérezTorres $^{12}$, M. Perri ${ }^{28}$, M. Persic ${ }^{103}$, S. Petrera ${ }^{18}$, P.-O. Petrucci ${ }^{125}$, O. Petruk ${ }^{66}$, B. Peyaud ${ }^{89}$, K. Pfrang ${ }^{52}$, E. Pian $^{21}$, G. Piano ${ }^{99}$, P. Piatteli ${ }^{94}$, E. Pietropaolo ${ }^{18}$, R. Pillera ${ }^{149}$, B. Pilszyk ${ }^{101}$, D. Pimentel ${ }^{202}$, F. Pintore ${ }^{91}$, C. Pio García ${ }^{41}$, G. Pirola ${ }^{64}$, F. Piron ${ }^{39}$, A. Pisarski ${ }^{190}$, S. Pita ${ }^{85}$, M. Pohl ${ }^{128}$, V. Poireau ${ }^{45}$, P. Poledrelli ${ }^{159}$, A. Pollo ${ }^{126}$, M. Polo ${ }^{113}$, C. Pongkitivanichkul ${ }^{31}$, J. Porthault ${ }^{144}$,

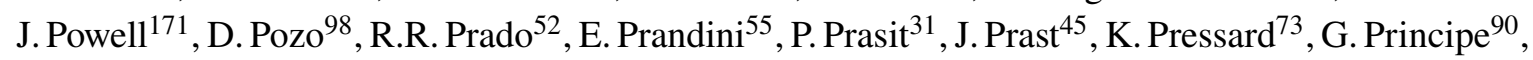
C. Priyadarshi ${ }^{41}$, N. Produit ${ }^{38}$, D. Prokhorov ${ }^{174}$, H. Prokoph ${ }^{52}$, M. Prouza ${ }^{33}$, H. Przybilski ${ }^{101}$, E. Pueschel ${ }^{52}$, G. Pühlhofer ${ }^{69}$, I. Puljak ${ }^{150}$, M.L. Pumo ${ }^{94}$, M. Punch ${ }^{85,57}$, F. Queiroz ${ }^{203}$, J. Quinn ${ }^{204}$, A. Quirrenbach ${ }^{170}$, S. Rainò ${ }^{149}$, P.J. Rajda ${ }^{175}$, R. Rando ${ }^{55}$, S. Razzaque ${ }^{205}$, E. Rebert ${ }^{20}$, S. Recchia ${ }^{85}$, P. Reichherzer ${ }^{59}$, O. Reimer ${ }^{163}$, A. Reimer ${ }^{163}$, A. Reisenegger ${ }^{3,206}$, Q. Remy ${ }^{53}$, M. Renaud ${ }^{39}$, T. Reposeur ${ }^{106}$, B. Reville ${ }^{53}$, J.-M. Reymond ${ }^{75}$, J. Reynolds ${ }^{15}$, W. Rhode ${ }^{46}$, D. Ribeiro ${ }^{9}$, M. Ribó ${ }^{81}$, G. Richards ${ }^{162}$, T. Richtler ${ }^{196}$, J. Rico ${ }^{41}$, F. Rieger ${ }^{53}$, L. Riitano ${ }^{135}$, V. Ripepi ${ }^{84}$, M. Riquelme ${ }^{192}$, D. Riquelme ${ }^{35}$, S. Rivoire ${ }^{39}$, V. Rizi ${ }^{18}$, E. Roache ${ }^{63}$, B. Röben ${ }^{159}$, M. Roche ${ }^{106}$, J. Rodriguez ${ }^{4}$, 
G. Rodriguez Fernandez ${ }^{25}$, J.C. Rodriguez Ramirez ${ }^{19}$, J.J. Rodríguez Vázquez ${ }^{113}$, F. Roepke ${ }^{170}$, G. $\operatorname{Rojas}^{207}$, L. Romanato ${ }^{55}$, P. Romano ${ }^{95}$, G. Romeo ${ }^{29}$, F. Romero Lobato ${ }^{11}$, C. Romoli ${ }^{53}$, M. Roncadelli1 ${ }^{103}$, S. Ronda ${ }^{30}$, J. Rosado ${ }^{11}$, A. Rosales de Leon ${ }^{5}$, G. Rowell ${ }^{118}$, B. Rudak ${ }^{49}$, A. Rugliancich ${ }^{74}$, J.E. Ruíz del Mazo ${ }^{12}$, W. Rujopakarn ${ }^{31}$, C. Rulten ${ }^{5}$, C. Russell ${ }^{3}$, F. Russo ${ }^{21}$, I. Sadeh ${ }^{52}$, E. Sæther Hatlen ${ }^{10}$, S. Safi-Harb ${ }^{37}$, L. Saha ${ }^{11}$, P. Saha ${ }^{208}$, V. Sahakian ${ }^{147}$, S. Sailer ${ }^{53}$, T. Saito ${ }^{2}$, N. Sakaki ${ }^{54}$, S. Sakurai ${ }^{2}$, F. Salesa Greus ${ }^{101}$, G. Salina ${ }^{25}$, H. Salzmann ${ }^{69}$, D. Sanchez ${ }^{45}$, M. Sánchez-Conde ${ }^{14}$, H. Sandaker ${ }^{10}$, A. Sandoval ${ }^{16}$, P. Sangiorgi ${ }^{91}$, M. Sanguillon ${ }^{39}$, H. Sano ${ }^{2}$, M. Santander ${ }^{171}$, A. Santangelo ${ }^{69}$, E.M. Santos ${ }^{202}$, R. Santos-Lima ${ }^{19}$, A. Sanuy ${ }^{81}$, L. Sapozhnikov ${ }^{96}$, T. Saric ${ }^{150}$, S. Sarkar ${ }^{14}$, H. Sasaki ${ }^{157}$, N. Sasaki ${ }^{179}$, K. Satalecka ${ }^{52}$, Y. Sato ${ }^{209}$, F.G. Saturni ${ }^{28}$, M. Sawada ${ }^{54}$, U. Sawangwit ${ }^{31}$, J. Schaefer ${ }^{142}$, A. Scherer ${ }^{3}$, J. Scherpenberg ${ }^{105}$, P. Schipani ${ }^{84}$, B. Schleicher ${ }^{122}$, J. Schmoll ${ }^{5}$, M. Schneider ${ }^{143}$, H. Schoorlemmer ${ }^{53}$, P. Schovanek ${ }^{33}$, F. Schussler ${ }^{89}$, B. Schwab ${ }^{142}$, U. Schwanke ${ }^{186}$, J. Schwarz ${ }^{95}$, T. Schweizer ${ }^{105}$, E. Sciacca ${ }^{29}$, S. Scuderi ${ }^{61}$, M. Seglar Arroyo $^{45}$, A. Segreto ${ }^{91}$, I. Seitenzahl ${ }^{43}$, D. Semikoz ${ }^{85}$, O. Sergijenko ${ }^{136}$, J.E. Serna Franco ${ }^{16}$, M. Servillat ${ }^{20}$, K. Seweryn ${ }^{201}$, V. Sguera ${ }^{21}$, A. Shalchi ${ }^{37}$, R.Y. Shang ${ }^{71}$, P. Sharma ${ }^{73}$, R.C. Shellard ${ }^{40}$, L. Sidoli ${ }^{61}$, J. Sieiro ${ }^{81}$, H. Siejkowski ${ }^{152}$, J. Silk ${ }^{114}$, A. Sillanpää ${ }^{65}$, B.B. Singh ${ }^{109}$, K.K. Singh ${ }^{210}$, A. Sinha ${ }^{39}$, C. Siqueira ${ }^{80}$, G. Sironi ${ }^{95}$, J. Sitarek ${ }^{60}$, P. Sizun ${ }^{75}$, V. Sliusar ${ }^{38}$, A. Slowikowska ${ }^{178}$, D. Sobczyńska ${ }^{60}$, R.W. Sobrinho ${ }^{184}$, H. Sol ${ }^{20}$, G. Sottile ${ }^{91}$, H. Spackman ${ }^{114}$, A. Specovius ${ }^{142}$, S. Spencer ${ }^{114}$, G. Spengler ${ }^{186}$, D. Spiga ${ }^{95}$, A. Spolon ${ }^{55}$, W. Springer ${ }^{164}$, A. Stamerra ${ }^{28}$, S. Stanič ${ }^{68}$, R. Starling ${ }^{124}$, L. Stawarz ${ }^{169}$, R. Steenkamp ${ }^{48}$, S. Stefanik ${ }^{197}$, C. Stegmann ${ }^{128}$, A. Steiner ${ }^{52}$, S. Steinmass ${ }^{53}$, C. Stella ${ }^{103}$, C. Steppa ${ }^{128}$, R. Sternberger ${ }^{52}$, M. Sterzel ${ }^{152}$, C. Stevens ${ }^{135}$, B. Stevenson ${ }^{71}$, T. Stolarczyk ${ }^{4}$, G. Stratta ${ }^{21}$, U. Straumann ${ }^{208}$, J. Strišković ${ }^{166}$, M. Strzys ${ }^{2}$, R. Stuik ${ }^{174}$, M. Suchenek ${ }^{211}$, Y. Suda ${ }^{140}$, Y. Sunada ${ }^{179}$, T. Suomijarvi ${ }^{73}$, T. Suric ${ }^{212}$, P. Sutcliffe ${ }^{153}$, H. Suzuki ${ }^{213}$, P. Świerk ${ }^{101}$, T. Szepieniec ${ }^{152}$, A. Tacchini ${ }^{21}$, K. Tachihara ${ }^{141}$, G. Tagliaferri ${ }^{95}$, H. Tajima ${ }^{139}$, N. Tajima ${ }^{2}$, D. Tak ${ }^{52}$, K. Takahashi ${ }^{214}$, H. Takahashi ${ }^{140}$, M. Takahashi ${ }^{2}$, M. Takahashi ${ }^{2}$, J. Takata ${ }^{2}$, R. Takeishi ${ }^{2}$, T. Tam ${ }^{2}$, M. Tanaka ${ }^{182}$, T. Tanaka ${ }^{213}$, S. Tanaka ${ }^{209}$, D. Tateishi ${ }^{179}$, M. Tavani ${ }^{99}$, F. Tavecchio ${ }^{95}$,

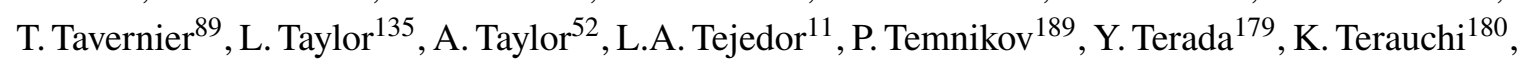
J.C. Terrazas ${ }^{192}$, R. Terrier ${ }^{85}$, T. Terzic ${ }^{121}$, M. Teshima ${ }^{105,2}$ V. Testa $^{28}$, D. Thibaut ${ }^{85}$, F. Thocquenne $^{75}$, W. Tian $^{2}$, L. Tibaldo ${ }^{87}$, A. Tiengo ${ }^{215}$, D. Tiziani ${ }^{142}$, M. Tluczykont ${ }^{50}$, C.J. Todero Peixoto ${ }^{102}$, F. Tokanai ${ }^{154}$, K. Toma ${ }^{160}$, L. Tomankova ${ }^{142}$, J. Tomastik ${ }^{104}$, D. Tonev ${ }^{189}$, M. Tornikoski ${ }^{216}$, D.F. Torres ${ }^{13}$, E. Torresi ${ }^{21}$, G. Tosti ${ }^{95}$, L. Tosti ${ }^{23}$, T. Totani ${ }^{51}$, N. Tothill ${ }^{117}$, F. Toussenel ${ }^{79}$,

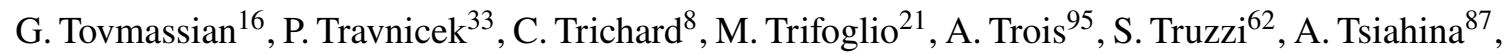

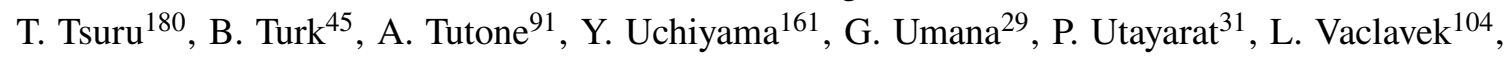
M. Vacula ${ }^{104}$, V. Vagelli ${ }^{23,217}$, F. Vagnetti ${ }^{25}$, F. Vakili $^{218}$, J.A. Valdivia ${ }^{192}$, M. Valentino ${ }^{24}$, A. Valio ${ }^{19}$, B. Vallage ${ }^{89}$, P. Vallania ${ }^{44,64}$, J.V. Valverde Quispe ${ }^{8}$, A.M. Van den Berg $^{42}$, W. van Driel ${ }^{20}$, C. van Eldik $^{142}$, C. van Rensburg ${ }^{1}$, B. van Soelen ${ }^{210}$, J. Vandenbroucke ${ }^{135}$, J. Vanderwalt ${ }^{1}$, G. Vasileiadis ${ }^{39}$, V. Vassiliev ${ }^{71}$, M. Vázquez Acosta ${ }^{32}$, M. Vecchi ${ }^{42}$, A. Vega ${ }^{98}$, J. Veh $^{142}$, P. Veitch ${ }^{118}$, P. Venault $^{75}$,

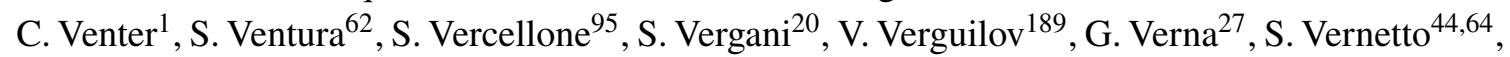
V. Verzi ${ }^{25}$, G.P. Vettolani ${ }^{90}$, C. Veyssiere ${ }^{144}$, I. Viale ${ }^{55}$, A. Viana ${ }^{80}$, N. Viaux ${ }^{35}$, J. Vicha ${ }^{33}$, J. Vignatti ${ }^{35}$, C.F. Vigorito ${ }^{64,108}$, J. Villanueva ${ }^{98}$, J. Vink ${ }^{174}$, V. Vitale ${ }^{23}$, V. Vittorinii ${ }^{99}$, V. Vodeb ${ }^{68}$, H. Voelk ${ }^{53}$, N. Vogel ${ }^{142}$, V. Voisin ${ }^{79}$, S. Vorobiov ${ }^{68}$, I. Vovk ${ }^{2}$, M. Vrastil ${ }^{33}$, T. Vuillaume ${ }^{45}$, S.J. Wagner ${ }^{170}$, R. Wagner ${ }^{105}$, P. Wagner ${ }^{52}$, K. Wakazono ${ }^{139}$, S.P. Wakely ${ }^{127}$, R. Walter ${ }^{38}$, M. Ward $^{5}$, D. Warren ${ }^{54}$, J. Watson ${ }^{52}$, N. Webb ${ }^{87}$, M. Wechakama ${ }^{31}$, P. Wegner ${ }^{52}$, A. Weinstein ${ }^{129}$, C. Weniger ${ }^{174}$, F. Werner ${ }^{53}$, H. Wetteskind ${ }^{105}$, M. White ${ }^{118}$, R. White ${ }^{53}$, A. Wierzcholska ${ }^{101}$, S. Wiesand ${ }^{52}$, 
R. Wijers ${ }^{174}$, M. Wilkinson ${ }^{124}$, M. Will ${ }^{105}$, D.A. Williams ${ }^{143}$, J. Williams ${ }^{124}$, T. Williamson ${ }^{162}$, A. Wolter ${ }^{95}$, Y.W. Wong ${ }^{142}$, M. Wood ${ }^{96}$, C. Wunderlich ${ }^{62}$, T. Yamamoto ${ }^{213}$, H. Yamamoto ${ }^{141}$,

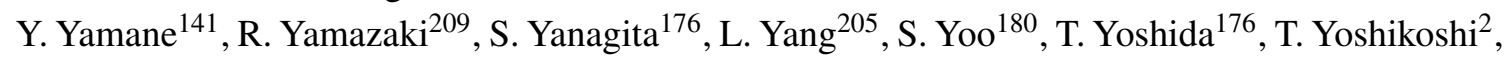
P. $\mathrm{Yu}^{71}$, P. Yu ${ }^{85}$, A. Yusafzaii ${ }^{59}$, M. Zacharias ${ }^{20}$, G. Zaharijas ${ }^{68}$, B. Zaldivar ${ }^{14}$, L. Zampieri ${ }^{76}$, R. Zanmar Sanchez ${ }^{29}$, D. Zaric ${ }^{150}$, M. Zavrtanik ${ }^{68}$, D. Zavrtanik ${ }^{68}$, A.A. Zdziarski ${ }^{49}$, A. Zech ${ }^{20}$, H.Zechlin ${ }^{64}$, A.Zenin ${ }^{139}$, A. Zerwekh ${ }^{35}$, V.I. Zhdanov ${ }^{136}$, K. Ziętara ${ }^{169}$, A. Zink ${ }^{142}$, J. Ziółkowski ${ }^{49}$, V. Zitelli2 ${ }^{21}$, M. Živec ${ }^{68}$, A. Zmija ${ }^{142}$

1: Centre for Space Research, North-West University, Potchefstroom, 2520, South Africa

2: Institute for Cosmic Ray Research, University of Tokyo, 5-1-5, Kashiwa-no-ha, Kashiwa, Chiba 277-8582, Japan

3 : Pontificia Universidad Católica de Chile, Av. Libertador Bernardo O’Higgins 340, Santiago, Chile

4 : AIM, CEA, CNRS, Université Paris-Saclay, Université Paris Diderot, Sorbonne Paris Cité, CEA Paris-Saclay, IRFU/DAp, Bat 709, Orme des Merisiers, 91191 Gif-sur-Yvette, France

5 : Centre for Advanced Instrumentation, Dept. of Physics, Durham University, South Road, Durham DH1 3LE, United Kingdom

6: Port d'Informació Científica, Edifici D, Carrer de l'Albareda, 08193 Bellaterrra (Cerdanyola del Vallès), Spain

7 : School of Physics and Astronomy, Monash University, Melbourne, Victoria 3800, Australia

8: Laboratoire Leprince-Ringuet, École Polytechnique (UMR 7638, CNRS/IN2P3, Institut Polytechnique de Paris), 91128 Palaiseau, France

9 : Department of Physics, Columbia University, 538 West 120th Street, New York, NY 10027, USA

10 : University of Oslo, Department of Physics, Sem Saelandsvei 24 - PO Box 1048 Blindern, N-0316 Oslo, Norway

11 : EMFTEL department and IPARCOS, Universidad Complutense de Madrid, 28040 Madrid, Spain

12 : Instituto de Astrofísica de Andalucía-CSIC, Glorieta de la Astronomía s/n, 18008, Granada, Spain

13 : Institute of Space Sciences (ICE-CSIC), and Institut d'Estudis Espacials de Catalunya (IEEC), and Institució Catalana de Recerca I Estudis Avançats (ICREA), Campus UAB, Carrer de Can Magrans, s/n 08193 Cerdanyola del Vallés, Spain

14 : Instituto de Física Teórica UAM/CSIC and Departamento de Física Teórica, Universidad Autónoma de Madrid, c/ Nicolás Cabrera 13-15, Campus de Cantoblanco UAM, 28049 Madrid, Spain

15 : Dublin Institute for Advanced Studies, 31 Fitzwilliam Place, Dublin 2, Ireland

16: Universidad Nacional Autónoma de México, Delegación Coyoacán, 04510 Ciudad de México, Mexico

17 : University of Geneva - Département de physique nucléaire et corpusculaire, 24 rue du GénéralDufour, 1211 Genève 4, Switzerland

18 : INFN Dipartimento di Scienze Fisiche e Chimiche - Università degli Studi dell'Aquila and Gran Sasso Science Institute, Via Vetoio 1, Viale Crispi 7, 67100 L'Aquila, Italy 
19 : Instituto de Astronomia, Geofísico, e Ciências Atmosféricas - Universidade de São Paulo, Cidade Universitária, R. do Matão, 1226, CEP 05508-090, São Paulo, SP, Brazil

20 : LUTH, GEPI and LERMA, Observatoire de Paris, CNRS, PSL University, 5 place Jules Janssen, 92190, Meudon, France

21 : INAF - Osservatorio di Astrofisica e Scienza dello spazio di Bologna, Via Piero Gobetti 93/3, 40129 Bologna, Italy

22 : INAF - Osservatorio Astrofisico di Arcetri, Largo E. Fermi, 5 - 50125 Firenze, Italy

23 : INFN Sezione di Perugia and Università degli Studi di Perugia, Via A. Pascoli, 06123 Perugia, Italy

24 : INFN Sezione di Napoli, Via Cintia, ed. G, 80126 Napoli, Italy

25 : INFN Sezione di Roma Tor Vergata, Via della Ricerca Scientifica 1, 00133 Rome, Italy

26 : Argonne National Laboratory, 9700 S. Cass Avenue, Argonne, IL 60439, USA

27 : Aix-Marseille Université, CNRS/IN2P3, CPPM, 163 Avenue de Luminy, 13288 Marseille cedex 09, France

28 : INAF - Osservatorio Astronomico di Roma, Via di Frascati 33, 00040, Monteporzio Catone, Italy

29: INAF - Osservatorio Astrofisico di Catania, Via S. Sofia, 78, 95123 Catania, Italy

30 : Grupo de Electronica, Universidad Complutense de Madrid, Av. Complutense s/n, 28040 Madrid, Spain

31 : National Astronomical Research Institute of Thailand, 191 Huay Kaew Rd., Suthep, Muang, Chiang Mai, 50200, Thailand

32 : Instituto de Astrofísica de Canarias and Departamento de Astrofísica, Universidad de La Laguna, La Laguna, Tenerife, Spain

33 : FZU - Institute of Physics of the Czech Academy of Sciences, Na Slovance 1999/2, 18221 Praha 8, Czech Republic

34 : Astronomical Institute of the Czech Academy of Sciences, Bocni II 1401 - 14100 Prague, Czech Republic

35 : CCTVal, Universidad Técnica Federico Santa María, Avenida España 1680, Valparaíso, Chile 36 : ETH Zurich, Institute for Particle Physics, Schafmattstr. 20, CH-8093 Zurich, Switzerland

37 : The University of Manitoba, Dept of Physics and Astronomy, Winnipeg, Manitoba R3T 2N2, Canada

38 : Department of Astronomy, University of Geneva, Chemin d'Ecogia 16, CH-1290 Versoix, Switzerland

39: Laboratoire Univers et Particules de Montpellier, Université de Montpellier, CNRS/IN2P3, CC 72, Place Eugène Bataillon, F-34095 Montpellier Cedex 5, France

40 : Centro Brasileiro de Pesquisas Físicas, Rua Xavier Sigaud 150, RJ 22290-180, Rio de Janeiro, Brazil

41 : Institut de Fisica d'Altes Energies (IFAE), The Barcelona Institute of Science and Technology, Campus UAB, 08193 Bellaterra (Barcelona), Spain

42 : University of Groningen, KVI - Center for Advanced Radiation Technology, Zernikelaan 25, 9747 AA Groningen, The Netherlands

43 : School of Physics, University of New South Wales, Sydney NSW 2052, Australia 
44 : INAF - Osservatorio Astrofisico di Torino, Strada Osservatorio 20, 10025 Pino Torinese (TO), Italy

45 : Univ. Savoie Mont Blanc, CNRS, Laboratoire d'Annecy de Physique des Particules - IN2P3, 74000 Annecy, France

46 : Department of Physics, TU Dortmund University, Otto-Hahn-Str. 4, 44221 Dortmund, Germany

47 : University of Zagreb, Faculty of electrical engineering and computing, Unska 3, 10000 Zagreb, Croatia

48 : University of Namibia, Department of Physics, 340 Mandume Ndemufayo Ave., Pioneerspark, Windhoek, Namibia

49 : Nicolaus Copernicus Astronomical Center, Polish Academy of Sciences, ul. Bartycka 18, 00-716 Warsaw, Poland

50 : Universität Hamburg, Institut für Experimentalphysik, Luruper Chaussee 149, 22761 Hamburg, Germany

51 : Graduate School of Science, University of Tokyo, 7-3-1 Hongo, Bunkyo-ku, Tokyo 113-0033, Japan

52 : Deutsches Elektronen-Synchrotron, Platanenallee 6, 15738 Zeuthen, Germany

53 : Max-Planck-Institut für Kernphysik, Saupfercheckweg 1, 69117 Heidelberg, Germany

54 : RIKEN, Institute of Physical and Chemical Research, 2-1 Hirosawa, Wako, Saitama, 351-0198, Japan

55 : INFN Sezione di Padova and Università degli Studi di Padova, Via Marzolo 8, 35131 Padova, Italy

56 : Escuela Politécnica Superior de Jaén, Universidad de Jaén, Campus Las Lagunillas s/n, Edif. A3, 23071 Jaén, Spain

57 : Department of Physics and Electrical Engineering, Linnaeus University, 35195 Växjö, Sweden

58 : University of the Witwatersrand, 1 Jan Smuts Avenue, Braamfontein, 2000 Johannesburg, South Africa

59 : Institut für Theoretische Physik, Lehrstuhl IV: Plasma-Astroteilchenphysik, Ruhr-Universität Bochum, Universitätsstraße 150, 44801 Bochum, Germany

60 : Faculty of Physics and Applied Computer Science, University of Lódź, ul. Pomorska 149-153, 90-236 Lódź, Poland

61 : INAF - Istituto di Astrofisica Spaziale e Fisica Cosmica di Milano, Via A. Corti 12, 20133 Milano, Italy

62: INFN and Università degli Studi di Siena, Dipartimento di Scienze Fisiche, della Terra e dell'Ambiente (DSFTA), Sezione di Fisica, Via Roma 56, 53100 Siena, Italy

63 : Center for Astrophysics | Harvard \& Smithsonian, 60 Garden St, Cambridge, MA 02180, USA

64 : INFN Sezione di Torino, Via P. Giuria 1, 10125 Torino, Italy

65 : Finnish Centre for Astronomy with ESO, University of Turku, Finland, FI-20014 University of Turku, Finland

66: Pidstryhach Institute for Applied Problems in Mechanics and Mathematics NASU, 3B Naukova Street, Lviv, 79060, Ukraine

67 : Bhabha Atomic Research Centre, Trombay, Mumbai 400085, India 
68 : Center for Astrophysics and Cosmology, University of Nova Gorica, Vipavska 11c, 5270 Ajdovščina, Slovenia

69 : Institut für Astronomie und Astrophysik, Universität Tübingen, Sand 1, 72076 Tübingen, Germany

70 : Research School of Astronomy and Astrophysics, Australian National University, Canberra ACT 0200, Australia

71 : Department of Physics and Astronomy, University of California, Los Angeles, CA 90095, USA

72: INFN Sezione di Bari and Politecnico di Bari, via Orabona 4, 70124 Bari, Italy

73: Laboratoire de Physique des 2 infinis, Irene Joliot-Curie,IN2P3/CNRS, Université Paris-Saclay, Université de Paris, 15 rue Georges Clemenceau, 91406 Orsay, Cedex, France

74 : INFN Sezione di Pisa, Largo Pontecorvo 3, 56217 Pisa, Italy

75 : IRFU/DEDIP, CEA, Université Paris-Saclay, Bat 141, 91191 Gif-sur-Yvette, France

76 : INAF - Osservatorio Astronomico di Padova, Vicolo dell'Osservatorio 5, 35122 Padova, Italy

77 : INAF - Osservatorio Astronomico di Palermo "G.S. Vaiana", Piazza del Parlamento 1, 90134 Palermo, Italy

78 : School of Physics, University of Sydney, Sydney NSW 2006, Australia

79: Sorbonne Université, Université Paris Diderot, Sorbonne Paris Cité, CNRS/IN2P3, Laboratoire de Physique Nucléaire et de Hautes Energies, LPNHE, 4 Place Jussieu, F-75005 Paris, France

80 : Instituto de Física de São Carlos, Universidade de São Paulo, Av. Trabalhador São-carlense, 400 - CEP 13566-590, São Carlos, SP, Brazil

81: Departament de Física Quàntica i Astrofísica, Institut de Ciències del Cosmos, Universitat de Barcelona, IEEC-UB, Martí i Franquès, 1, 08028, Barcelona, Spain

82 : Department of Physics, Washington University, St. Louis, MO 63130, USA

83 : Saha Institute of Nuclear Physics, Bidhannagar, Kolkata-700 064, India

84 : INAF - Osservatorio Astronomico di Capodimonte, Via Salita Moiariello 16, 80131 Napoli, Italy

85 : Université de Paris, CNRS, Astroparticule et Cosmologie, 10, rue Alice Domon et Léonie Duquet, 75013 Paris Cedex 13, France

86 : Astronomy Department of Faculty of Physics, Sofia University, 5 James Bourchier Str., 1164 Sofia, Bulgaria

87: Institut de Recherche en Astrophysique et Planétologie, CNRS-INSU, Université Paul Sabatier, 9 avenue Colonel Roche, BP 44346, 31028 Toulouse Cedex 4, France

88 : School of Physics and Astronomy, University of Minnesota, 116 Church Street S.E. Minneapolis, Minnesota 55455-0112, USA

89 : IRFU, CEA, Université Paris-Saclay, Bât 141, 91191 Gif-sur-Yvette, France

90 : INAF - Istituto di Radioastronomia, Via Gobetti 101, 40129 Bologna, Italy

91 : INAF - Istituto di Astrofisica Spaziale e Fisica Cosmica di Palermo, Via U. La Malfa 153, 90146 Palermo, Italy

92 : Astronomical Observatory, Department of Physics, University of Warsaw, Aleje Ujazdowskie 4, 00478 Warsaw, Poland

93 : Armagh Observatory and Planetarium, College Hill, Armagh BT61 9DG, United Kingdom

94 : INFN Sezione di Catania, Via S. Sofia 64, 95123 Catania, Italy

95 : INAF - Osservatorio Astronomico di Brera, Via Brera 28, 20121 Milano, Italy 
96 : Kavli Institute for Particle Astrophysics and Cosmology, Department of Physics and SLAC National Accelerator Laboratory, Stanford University, 2575 Sand Hill Road, Menlo Park, CA 94025, USA

97 : Universidade Cruzeiro do Sul, Núcleo de Astrofísica Teórica (NAT/UCS), Rua Galvão Bueno 8687, Bloco B, sala 16, Libertade 01506-000 - São Paulo, Brazil

98 : Universidad de Valparaíso, Blanco 951, Valparaiso, Chile

99: INAF - Istituto di Astrofisica e Planetologia Spaziali (IAPS), Via del Fosso del Cavaliere 100, 00133 Roma, Italy

100 : Lund Observatory, Lund University, Box 43, SE-22100 Lund, Sweden

101 : The Henryk Niewodniczański Institute of Nuclear Physics, Polish Academy of Sciences, ul. Radzikowskiego 152, 31-342 Cracow, Poland

102 : Escola de Engenharia de Lorena, Universidade de São Paulo, Área I - Estrada Municipal do Campinho, s/nº, CEP 12602-810, Pte. Nova, Lorena, Brazil

103 : INFN Sezione di Trieste and Università degli Studi di Udine, Via delle Scienze 208, 33100 Udine, Italy

104 : Palacky University Olomouc, Faculty of Science, RCPTM, 17. listopadu 1192/12, 77146 Olomouc, Czech Republic

105 : Max-Planck-Institut für Physik, Föhringer Ring 6, 80805 München, Germany

106 : CENBG, Univ. Bordeaux, CNRS-IN2P3, UMR 5797, 19 Chemin du Solarium, CS 10120, F-33175 Gradignan Cedex, France

107 : Dublin City University, Glasnevin, Dublin 9, Ireland

108 : Dipartimento di Fisica - Universitá degli Studi di Torino, Via Pietro Giuria 1 - 10125 Torino, Italy

109: Tata Institute of Fundamental Research, Homi Bhabha Road, Colaba, Mumbai 400005, India 110: Universitá degli Studi di Napoli "Federico II" - Dipartimento di Fisica "E. Pancini", Complesso universitario di Monte Sant'Angelo, Via Cintia - 80126 Napoli, Italy

111 : Oskar Klein Centre, Department of Physics, University of Stockholm, Albanova, SE-10691, Sweden

112 : Yale University, Department of Physics and Astronomy, 260 Whitney Avenue, New Haven, CT 06520-8101, USA

113 : CIEMAT, Avda. Complutense 40, 28040 Madrid, Spain

114 : University of Oxford, Department of Physics, Denys Wilkinson Building, Keble Road, Oxford OX1 3RH, United Kingdom

115 : School of Physics \& Astronomy, University of Southampton, University Road, Southampton SO17 1BJ, United Kingdom

116 : Department of Physics and Technology, University of Bergen, Museplass 1, 5007 Bergen, Norway

117 : Western Sydney University, Locked Bag 1797, Penrith, NSW 2751, Australia

118: School of Physical Sciences, University of Adelaide, Adelaide SA 5005, Australia

119 : INFN Sezione di Roma La Sapienza, P.le Aldo Moro, 2 - 00185 Roma, Italy

120 : INFN Sezione di Bari, via Orabona 4, 70126 Bari, Italy

121 : University of Rijeka, Department of Physics, Radmile Matejcic 2, 51000 Rijeka, Croatia 
122 : Institute for Theoretical Physics and Astrophysics, Universität Würzburg, Campus Hubland Nord, Emil-Fischer-Str. 31, 97074 Würzburg, Germany

123 : Universidade Federal Do Paraná - Setor Palotina, Departamento de Engenharias e Exatas, Rua Pioneiro, 2153, Jardim Dallas, CEP: 85950-000 Palotina, Paraná, Brazil

124 : Dept. of Physics and Astronomy, University of Leicester, Leicester, LE1 7RH, United Kingdom

125 : Univ. Grenoble Alpes, CNRS, IPAG, 414 rue de la Piscine, Domaine Universitaire, 38041 Grenoble Cedex 9, France

126 : National Centre for nuclear research (Narodowe Centrum Badań Jądrowych), Ul. Andrzeja Sołtana7, 05-400 Otwock, Świerk, Poland

127 : Enrico Fermi Institute, University of Chicago, 5640 South Ellis Avenue, Chicago, IL 60637, USA

128: Institut für Physik \& Astronomie, Universität Potsdam, Karl-Liebknecht-Strasse 24/25, 14476 Potsdam, Germany

129 : Department of Physics and Astronomy, Iowa State University, Zaffarano Hall, Ames, IA 50011-3160, USA

130 : School of Physics, Aristotle University, Thessaloniki, 54124 Thessaloniki, Greece

131 : King's College London, Strand, London, WC2R 2LS, United Kingdom

132 : Escola de Artes, Ciências e Humanidades, Universidade de São Paulo, Rua Arlindo Bettio, CEP 03828-000, 1000 São Paulo, Brazil

133 : Dept. of Astronomy \& Astrophysics, Pennsylvania State University, University Park, PA 16802, USA

134 : National Technical University of Athens, Department of Physics, Zografos 9, 15780 Athens, Greece

135 : University of Wisconsin, Madison, 500 Lincoln Drive, Madison, WI, 53706, USA

136: Astronomical Observatory of Taras Shevchenko National University of Kyiv, 3 Observatorna Street, Kyiv, 04053, Ukraine

137 : Department of Physics, Purdue University, West Lafayette, IN 47907, USA

138 : Unitat de Física de les Radiacions, Departament de Física, and CERES-IEEC, Universitat Autònoma de Barcelona, Edifici C3, Campus UAB, 08193 Bellaterra, Spain

139 : Institute for Space-Earth Environmental Research, Nagoya University, Chikusa-ku, Nagoya 464-8601, Japan

140 : Department of Physical Science, Hiroshima University, Higashi-Hiroshima, Hiroshima 7398526, Japan

141 : Department of Physics, Nagoya University, Chikusa-ku, Nagoya, 464-8602, Japan

142: Friedrich-Alexander-Universität Erlangen-Nürnberg, Erlangen Centre for Astroparticle Physics (ECAP), Erwin-Rommel-Str. 1, 91058 Erlangen, Germany

143 : Santa Cruz Institute for Particle Physics and Department of Physics, University of California, Santa Cruz, 1156 High Street, Santa Cruz, CA 95064, USA

144 : IRFU / DIS, CEA, Université de Paris-Saclay, Bat 123, 91191 Gif-sur-Yvette, France

145 : INFN Sezione di Trieste and Università degli Studi di Trieste, Via Valerio 2 I, 34127 Trieste, Italy 
146 : School of Physics \& Center for Relativistic Astrophysics, Georgia Institute of Technology, 837 State Street, Atlanta, Georgia, 30332-0430, USA

147 : Alikhanyan National Science Laboratory, Yerevan Physics Institute, 2 Alikhanyan Brothers St., 0036, Yerevan, Armenia

148 : INAF - Telescopio Nazionale Galileo, Roche de los Muchachos Astronomical Observatory, 38787 Garafia, TF, Italy

149 : INFN Sezione di Bari and Università degli Studi di Bari, via Orabona 4, 70124 Bari, Italy

150 : University of Split - FESB, R. Boskovica 32, 21000 Split, Croatia

151: Universidad Andres Bello, República 252, Santiago, Chile

152: Academic Computer Centre CYFRONET AGH, ul. Nawojki 11, 30-950 Cracow, Poland

153 : University of Liverpool, Oliver Lodge Laboratory, Liverpool L69 7ZE, United Kingdom

154 : Department of Physics, Yamagata University, Yamagata, Yamagata 990-8560, Japan

155 : Astronomy Department, Adler Planetarium and Astronomy Museum, Chicago, IL 60605, USA

156 : Faculty of Management Information, Yamanashi-Gakuin University, Kofu, Yamanashi 4008575, Japan

157 : Department of Physics, Tokai University, 4-1-1, Kita-Kaname, Hiratsuka, Kanagawa 2591292, Japan

158 : Centre for Astrophysics Research, Science \& Technology Research Institute, University of Hertfordshire, College Lane, Hertfordshire AL10 9AB, United Kingdom

159: Cherenkov Telescope Array Observatory, Saupfercheckweg 1, 69117 Heidelberg, Germany

160 : Tohoku University, Astronomical Institute, Aobaku, Sendai 980-8578, Japan

161 : Department of Physics, Rikkyo University, 3-34-1 Nishi-Ikebukuro, Toshima-ku, Tokyo, Japan

162 : Department of Physics and Astronomy and the Bartol Research Institute, University of Delaware, Newark, DE 19716, USA

163 : Institut für Astro- und Teilchenphysik, Leopold-Franzens-Universität, Technikerstr. 25/8, 6020 Innsbruck, Austria

164 : Department of Physics and Astronomy, University of Utah, Salt Lake City, UT 84112-0830, USA

165 : IMAPP, Radboud University Nijmegen, P.O. Box 9010, 6500 GL Nijmegen, The Netherlands 166 : Josip Juraj Strossmayer University of Osijek, Trg Ljudevita Gaja 6, 31000 Osijek, Croatia

167 : Department of Earth and Space Science, Graduate School of Science, Osaka University, Toyonaka 560-0043, Japan

168: Yukawa Institute for Theoretical Physics, Kyoto University, Kyoto 606-8502, Japan

169 : Astronomical Observatory, Jagiellonian University, ul. Orla 171, 30-244 Cracow, Poland

170 : Landessternwarte, Zentrum für Astronomie der Universität Heidelberg, Königstuhl 12, 69117 Heidelberg, Germany

171 : University of Alabama, Tuscaloosa, Department of Physics and Astronomy, Gallalee Hall, Box 870324 Tuscaloosa, AL 35487-0324, USA

172: Department of Physics, University of Bath, Claverton Down, Bath BA2 7AY, United Kingdom 173 : University of Iowa, Department of Physics and Astronomy, Van Allen Hall, Iowa City, IA 52242, USA 
174 : Anton Pannekoek Institute/GRAPPA, University of Amsterdam, Science Park 9041098 XH Amsterdam, The Netherlands

175 : Faculty of Computer Science, Electronics and Telecommunications, AGH University of Science and Technology, Kraków, al. Mickiewicza 30, 30-059 Cracow, Poland

176 : Faculty of Science, Ibaraki University, Mito, Ibaraki, 310-8512, Japan

177 : Faculty of Science and Engineering, Waseda University, Shinjuku, Tokyo 169-8555, Japan

178 : Institute of Astronomy, Faculty of Physics, Astronomy and Informatics, Nicolaus Copernicus University in Toruń, ul. Grudziądzka 5, 87-100 Toruń, Poland

179: Graduate School of Science and Engineering, Saitama University, 255 Simo-Ohkubo, Sakuraku, Saitama city, Saitama 338-8570, Japan

180 : Division of Physics and Astronomy, Graduate School of Science, Kyoto University, Sakyo-ku, Kyoto, 606-8502, Japan

181 : Centre for Quantum Technologies, National University Singapore, Block S15, 3 Science Drive 2, Singapore 117543, Singapore

182 : Institute of Particle and Nuclear Studies, KEK (High Energy Accelerator Research Organization), 1-1 Oho, Tsukuba, 305-0801, Japan

183 : Department of Physics and Astronomy, University of Sheffield, Hounsfield Road, Sheffield S3 7RH, United Kingdom

184 : Centro de Ciências Naturais e Humanas, Universidade Federal do ABC, Av. dos Estados, 5001, CEP: 09.210-580, Santo André - SP, Brazil

185 : Dipartimento di Fisica e Astronomia, Sezione Astrofisica, Universitá di Catania, Via S. Sofia 78, I-95123 Catania, Italy

186 : Department of Physics, Humboldt University Berlin, Newtonstr. 15, 12489 Berlin, Germany

187 : Texas Tech University, 2500 Broadway, Lubbock, Texas 79409-1035, USA

188 : University of Zielona Góra, ul. Licealna 9, 65-417 Zielona Góra, Poland

189 : Institute for Nuclear Research and Nuclear Energy, Bulgarian Academy of Sciences, 72 boul. Tsarigradsko chaussee, 1784 Sofia, Bulgaria

190 : University of Białystok, Faculty of Physics, ul. K. Ciołkowskiego 1L, 15-254 Białystok, Poland

191 : Faculty of Physics, National and Kapodestrian University of Athens, Panepistimiopolis, 15771 Ilissia, Athens, Greece

192 : Universidad de Chile, Av. Libertador Bernardo O'Higgins 1058, Santiago, Chile

193 : Hiroshima Astrophysical Science Center, Hiroshima University, Higashi-Hiroshima, Hiroshima 739-8526, Japan

194 : Department of Applied Physics, University of Miyazaki, 1-1 Gakuen Kibana-dai Nishi, Miyazaki, 889-2192, Japan

195 : School of Allied Health Sciences, Kitasato University, Sagamihara, Kanagawa 228-8555, Japan

196 : Departamento de Astronomía, Universidad de Concepción, Barrio Universitario S/N, Concepción, Chile

197 : Charles University, Institute of Particle \& Nuclear Physics, V Holešovičkách 2, 18000 Prague

8, Czech Republic 
198: Astronomical Observatory of Ivan Franko National University of Lviv, 8 Kyryla i Mephodia Street, Lviv, 79005, Ukraine

199 : Kobayashi-Maskawa Institute (KMI) for the Origin of Particles and the Universe, Nagoya University, Chikusa-ku, Nagoya 464-8602, Japan

200 : Graduate School of Technology, Industrial and Social Sciences, Tokushima University, Tokushima 770-8506, Japan

201 : Space Research Centre, Polish Academy of Sciences, ul. Bartycka 18A, 00-716 Warsaw, Poland

202 : Instituto de Física - Universidade de São Paulo, Rua do Matão Travessa R Nr.187 CEP 05508-090 Cidade Universitária, São Paulo, Brazil

203 : International Institute of Physics at the Federal University of Rio Grande do Norte, Campus Universitário, Lagoa Nova CEP 59078-970 Rio Grande do Norte, Brazil

204 : University College Dublin, Belfield, Dublin 4, Ireland

205 : Centre for Astro-Particle Physics (CAPP) and Department of Physics, University of Johannesburg, PO Box 524, Auckland Park 2006, South Africa

206: Departamento de Física, Facultad de Ciencias Básicas, Universidad Metropolitana de Ciencias de la Educación, Santiago, Chile

207 : Núcleo de Formação de Professores - Universidade Federal de São Carlos, Rodovia Washington Luís, km 235 CEP 13565-905 - SP-310 São Carlos - São Paulo, Brazil

208 : Physik-Institut, Universität Zürich, Winterthurerstrasse 190, 8057 Zürich, Switzerland

209 : Department of Physical Sciences, Aoyama Gakuin University, Fuchinobe, Sagamihara, Kanagawa, 252-5258, Japan

210 : University of the Free State, Nelson Mandela Avenue, Bloemfontein, 9300, South Africa

211 : Faculty of Electronics and Information, Warsaw University of Technology, ul. Nowowiejska 15/19, 00-665 Warsaw, Poland

212 : Rudjer Boskovic Institute, Bijenicka 54, 10000 Zagreb, Croatia

213 : Department of Physics, Konan University, Kobe, Hyogo, 658-8501, Japan

214 : Kumamoto University, 2-39-1 Kurokami, Kumamoto, 860-8555, Japan

215 : University School for Advanced Studies IUSS Pavia, Palazzo del Broletto, Piazza della Vittoria 15, 27100 Pavia, Italy

216: Aalto University, Otakaari 1, 00076 Aalto, Finland

217 : Agenzia Spaziale Italiana (ASI), 00133 Roma, Italy

218: Observatoire de la Cote d'Azur, Boulevard de l'Observatoire CS34229, 06304 Nice Cedex 4, Franc 